\title{
Flexoelectric materials and their related applications: A focused review
}

\author{
Longlong $\mathrm{SHU}^{a, b, *}$, Renhong $\mathrm{LIANG}^{a}$, Zhenggang RAO ${ }^{a, b}$, \\ Linfeng $\mathrm{FEI}^{a, b}$, Shanming $\mathrm{KE}^{a, b, *}$, Yu WANG ${ }^{a, b}$ \\ ${ }^{a}$ School of Materials Science and Engineering, Nanchang University, Nanchang 330031, China \\ ${ }^{b}$ Jiangxi Key Laboratory for Two-dimensional Materials and Devices, and Jiangxi Engineering \\ Laboratory for Advanced Functional Thin Films, Nanchang University, Nanchang 330031, China
}

Received: December 5, 2018; Accepted: December 8, 2018

(C) The Author(s) 2019.

\begin{abstract}
Flexoelectricity refers to the mechanical-electro coupling between strain gradient and electric polarization, and conversely, the electro-mechanical coupling between electric field gradient and mechanical stress. This unique effect shows a promising size effect which is usually large as the material dimension is shrunk down. Moreover, it could break the limitation of centrosymmetry, and has been found in numerous kinds of materials which cover insulators, liquid crystals, biological materials, and semiconductors. In this review, we will give a brief report about the recent discoveries in flexoelectricity, focusing on the flexoelectric materials and their applications. The theoretical developments in this field are also addressed. In the end, the perspective of flexoelectricity and some open questions which still remain unsolved are commented upon.

Keywords: flexoelectricity; strain gradient; electric polarization; dielectric constant; liquid crystals; sensors and actuators
\end{abstract}

\section{General concept of flexoelectricity}

Materials are usually classified into insulators, semiconductors, and conductors according to their different conduction modes in response to the external electric field. Specifically, the insulators transfer the function of external electric field by inducting rather than conducting. Quite recently a universal physical phenomenon, namely flexoelectricity, has rekindled considerable research interest in insulators due to its great application potential in many fields, e.g., new types of memory, domain engineering, defect tailoring, and non-piezoelectric sensors and actuators [1-4]. This

* Corresponding authors.

E-mail: L. Shu, 1lshu@ncu.edu.cn; S. Ke, ksm@ncu.edu.cn phenomenon describes the mechanical-electro coupling between the strain gradient and the electric polarization (direct flexoelectric effect), or vice versa, the coupling between the electric field gradient and the stress (converse flexoelectric effect), which can be written as [5]:

$$
P_{l}=\mu_{i j k l} \frac{\partial S_{i j}}{\partial x_{k}}, T_{i j}=\mu_{i j k l} \frac{\partial E_{k}}{\partial x_{l}}
$$

where $P_{l}$ is the induced polarization, $T_{i j}$ is the induced stress, $S_{i j}$ is the strain, and $E_{k}$ is the electric field, $x$ is the axis of coordinate, $\mu_{i j k l}$ is the direct flexoelectric coefficient with respect to strain gradient.

The concept of flexoelectricity was firstly originated from liquid crystals [6]. In liquid crystals, flexoelectricity refers to the reorientation of irregularly shaped polarized molecules under strain gradients 
caused by splay-deformations or bent-deformations, which is different from piezoelectric effect (produced by uniform strain or stress) $[7,8]$. In some soft materials like hairs and bio-membranes, similar phenomenon that the net charges between the internal and external surface of the material occur in response to the different curvature radiuses was also found $[9,10]$.

As a distinctive mechanical-electro effect, flexoelectricity has two main attractive features. (i) The flexoelectric coefficient is a fourth rank tensor, therefore flexoelectricity could break the limitation of crystal symmetry, and exist in those materials with centrosymmetry, which is quite different from the piezoelectric effect [11-13]. (ii) Strain gradient is inversely proportional to the material size, making the nanoscale flexoelectricity extremely large [14-16]. Such characteristic is called size effect or scaling effect, and has already been utilized in many nanostructures for sensing and actuating applications $[17,18]$.

\section{Flexoelectricity in materials}

\section{1 Direct and indirect measurement of flexoelectric coefficients}

Although the flexoelectric coupling should be principally existed in all insulators, studies on exploring the flexoelectric coefficient in specific materials are still quite limited. This could be explained by the following two reasons.

(i) In most of the dielectrics, the typical value of the flexoelectric coefficient is only in the range of $0.01-1$ $\mathrm{nC} / \mathrm{m}$. This characteristic value is generally regarded as an intrinsic flexoelectric coefficient. In 1986, Tagantsev proposed that the flexoelectric coefficients in general materials are comparable to their $e / a$ value $[19,20]$, where $e$ is the electron charge and $a$ is the lattice parameter. This theoretical value is in good accordance with the recent first principle calculations developed by Maranganti and Sharma [21] and Hong and Vanderbilt $[22,23]$ in some semiconductors and ferroelectric perovskites (as shown in Table 1). Note that the unit $\mathrm{C} / \mathrm{m}$ is quite different with the unit of piezoelectric coefficients $(\mathrm{C} / \mathrm{N})$. Therefore, it is inappropriate to make a direct comparison between flexoelectricity and piezoelectricity, just by using the numerical value of flexoelectric coefficients with that of the piezoelectric coefficients. Recent studies by Abdollahi et al. [24] suggested that $1 \mathrm{pC} / \mathrm{N}$ of piezoelectric coefficient is comparable to $10^{3} \mathrm{nC} / \mathrm{m}$ of flexoelectric coefficient. Based on this result, we can conclude that the flexoelectric coupling in most of the dielectrics is much weaker than the piezoelectric one.

(ii) The term "strain gradient" generally leads to very sophisticated tensor components of flexoelectric coefficient and then makes their measurements very difficult. According to the early studies by Le Quang and $\mathrm{He}$ [25] and Shu et al. [12], in a material with triclinic symmetry, the possible components of flexoelectric coefficients $\mu_{i j k l}$ could reach to a number as high as 54. Therefore, it is almost impossible to precisely extract individual flexoelectric components in those low-symmetry systems. On the other hand, in the materials with cubic symmetry (except for the 23 and $m 3$ point group symmetries), the components of flexoelectric tensor $\mu_{i j k l}$ could be reduced to only 3 , which is generally defined as the transverse flexoelectric coefficient, longitudinal flexoelectric coefficient, and shear flexoelectric coefficient.

During the past few years, three direct measurement methods have been developed by using the cantilever beam (Fig. 1(a)), the bottom-up side of truncated pyramid (Fig. 1(b)), and the lateral side of truncated pyramid (Fig. 1(c)) to measure the transverse flexoelectric coefficient, longitudinal flexoelectric coefficient, and shear flexoelectric coefficient, respectively $[5,26]$. It is worth noting that all the measurement setups in Fig. 1 are not highly integrated, and therefore the determination of all the aforementioned flexoelectric components is highly constrained.

In addition to these direct measurements, a few indirect measurements of the flexoelectric coefficients have been recently developed. Zhou et al. [27] found that the flexoelectric coefficients could be indirectly evaluated by using the variation of the stiffness. It is because the flexoelectricity will result in a rapid decline of the stiffness of a dielectric nano-conefrustum, especially at small size. The detailed principle and structure are described in Fig. 2(a). By studying the nanocompression force vs. the nanocompression displacement curves of the column and cone-frustum samples (as shown in Fig. 2(b)), the longitudinal flexoelectric coefficient could be successfully extracted. $\mathrm{Hu}$ et al. [28] proposed a special way to generate strain gradient by using a shock wave. In their experiment, the first-order hydrogen gas gun was employed to fire a flying plate and hit the non-polarized bulk $\mathrm{BaTiO}_{3}$ 


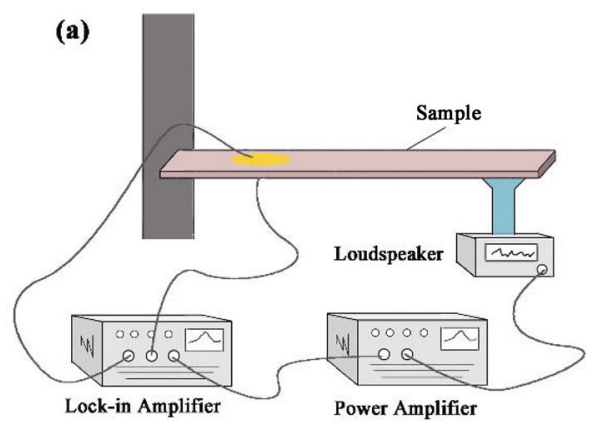

(b)

(c)

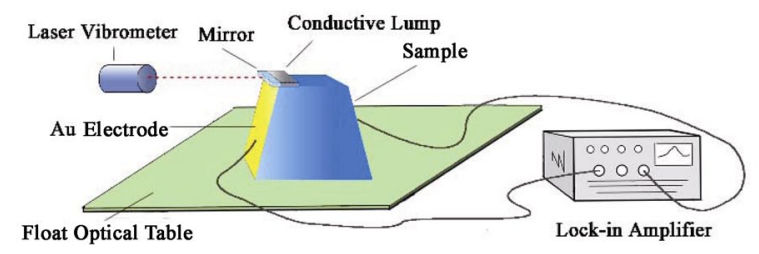

Fig. 1 Experimental setup for measuring three flexoelectric components. (a) The cantilever beam system which corresponds to the transverse flexoelectric coefficients. The measurement mechanism is by bending the cantilever sample in order to form a strain gradient $\partial S_{11} / \partial x_{3}$ and measuring the induced polarization simultaneously. (b) The measuring system of the longitudinal flexoelectric coefficients. The measurement mechanism is by applying a mechanical force to the truncated pyramid sample in order to form a strain gradient $\partial S_{33} / \partial x_{3}$ and measuring the induced polarization $P_{3}$ simultaneously. (c) The measuring system of the shear flexoelectric coefficients. The measurement mechanism is by applying voltage to the lateral side of the truncated pyramid sample in order to generate the electric field gradient $\partial E_{1} / \partial x_{3}$ and measuring the induced shear strain $S_{13}$ simultaneously.

(a)

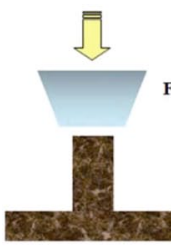

Constant Cross-section Sample Variable Cross-section Sample

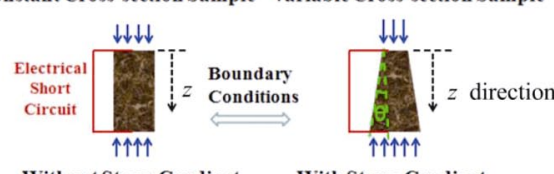

Without Stress Gradient ～With Stress Gradient

Flying Plate Gold Electrodes

(c)

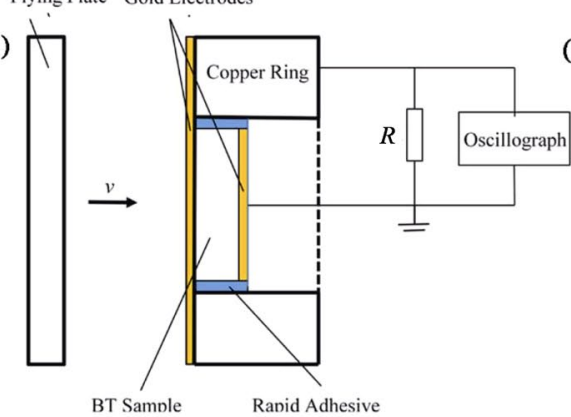

(b)

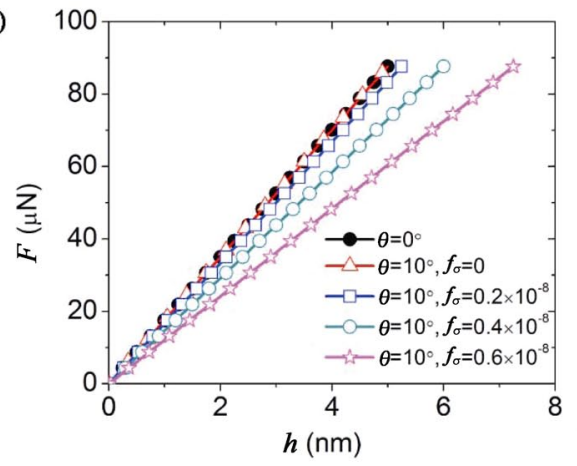

(d)

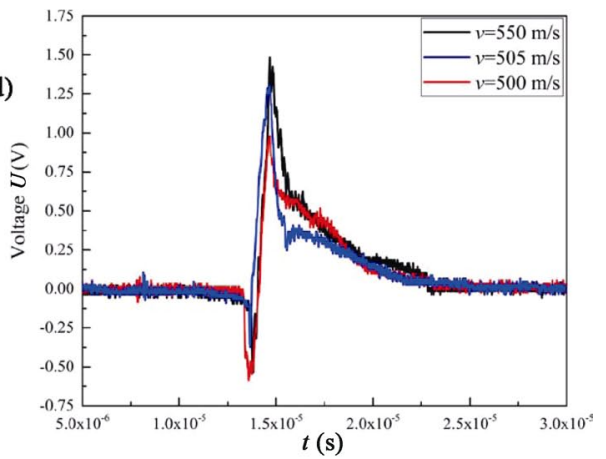

Fig. 2 (a) Schematic diagram of the nanocompression measurement. In principle, the nanocompression causes homogeneous stress in the constant cross-section pillar, but it will induce stress gradient in the longitudinal direction of the variable cross-section pillar. (b) The nanocompression force as a function of the nanocompression displacement curves of the column and cone-frustum samples with various flexoelectric coupling coefficient. Reproduced with permission from Ref. [27], (C) AIP Publishing 2016. (c) Schematic view of the one-dimensional shock wave setup for measuring the flexoelectric coefficients. (d) The induced voltage of bulk $\mathrm{BaTiO}_{3}$ samples as a function of shock waves. As the shock wave propagates into the sample, the negative voltage changes to positive voltage, and its value increases rapidly due to the flexoelectric effect which is caused by the strain gradient. Reproduced with permission from Ref. [28], (c) AIP Publishing 2017. 
ceramic (Fig. 2(c)). Through this method, the longitudinal flexoelectric coefficient could be indirectly calculated by studying the relationship between the induced voltage and the propagation of the shock waves, as shown in Fig. 2(d).

\section{2 Flexoelectric coefficients in dielectrics and ferroelectrics}

With the help of those direct and indirect measurements, a few polymers and dielectrics have been employed for studying their flexoelectric coefficients. As shown in Table 1, it is proved that the flexoelectric coefficients in normal dielectrics and polymers, represented by $\mathrm{TiO}_{2}$ ceramic and the polyvinylidene fluoride (PVDF) [29-34], are in the range of $1-10 \mathrm{nC} / \mathrm{m}$, which is in good accordance with the intrinsic flexoelectric values. But in some kinds of ferroelectric ceramics and single crystals, the results are quite different. Extremely enhanced flexoelectricity was found in a series of high permittivity ferroelectrics such as $\mathrm{BaTiO}_{3}$ (BT) [35,36], (BaSr)TiO ${ }_{3}$ (BST) [37-39], $\mathrm{Ba}(\mathrm{TiSn}) \mathrm{O}_{3}$ (BTS) [40,41], $\mathrm{Pb}\left(\mathrm{ZrTi}_{3} \mathrm{O}_{3}\right.$ (PZT) [42], (KNa) $\mathrm{NbO}_{3}$ [43], and $\mathrm{Pb}\left(\mathrm{Mg}_{1 / 3} \mathrm{Nb}_{2 / 3}\right) \mathrm{O}_{3}$ based ferroelectrics [44-46]. Their measured flexoelectric coefficients are 3-5 orders of magnitude larger than that in normal dielectrics such as $\mathrm{TiO}_{2}$. Intriguingly, such enhancement is not a universal phenomenon in ferroelectrics. For the incipient ferroelectric $\mathrm{SrTiO}_{3}$ and antiferroelectric $\mathrm{PbZrO}_{3}$, the transverse flexoelectric coefficient is only $1-2 \mathrm{nC} / \mathrm{m}$, which is just comparable with the normal dielectrics $[47,48]$.

In very recent years, the physical mechanism of the enhanced flexoelectricity in some dielectrics was intensively discussed [5,24,49-54]. The possible mechanisms are summarized in Fig. 3. The state-ofthe-art interpretation is not unified and much more work is urgently needed. Herein we want to emphasize that the extremely sensitive unit of the flexoelectric coefficients and their un-integrated electrical measurement may make an interference for the understanding of flexoelectricity. In real finite materials, all the factors, e.g., inner micro strain, polar nanoregions, and surface piezoelectricity, could be easily coupled into the flexoelectric coefficients. Therefore, the observed flexoelectric coefficients in dielectrics should be a competition/combination of many factors. It is noticed that the recent studies by Zhang et al. [54] suggested that the surface piezoelectricity can contribute about $70 \%$ of the enhanced flexoelectricity in $\mathrm{BaTiO}_{3}$ ceramics, and hence revealed this factor might be dominant in many other ferroelectrics where the observed flexoelectric coefficients were significantly enhanced.

\section{3 Flexoelectricity in biological materials}

Recent studies suggest that many biological materials such as bones, hairs, and bio-membranes have remarkable flexoelectric response [55-59]. The first studies of the flexoelectricity in biological materials could be traced back to 1975 by Williams and Breger [60]. In this initial study, some of the electro-mechanical properties of bones were considered to be likely originated from "gradient polarization", but the mechanism is not clear yet. To clarify the origin of this electro-mechanical property in bones, Vasquez-Sancho et al. [61] compared the flexoelectricity of bone and pure hydroxyapatite (which is the main mineral of bone) by using the traditional cantilever system. As shown in Fig. 4(a), the measured flexoelectricity in both bone and pure hydroxyapatite was found to be of the same order of magnitude, suggesting that the hydroxyapatite flexoelectricity is the main source of the bendinginduced polarization in bones. Furthermore, the result also revealed that the flexoelectricity in hydroxyapatite is helpful to the bone repair and remodeling processes. It is because that, as shown in Fig. 4(b), a large

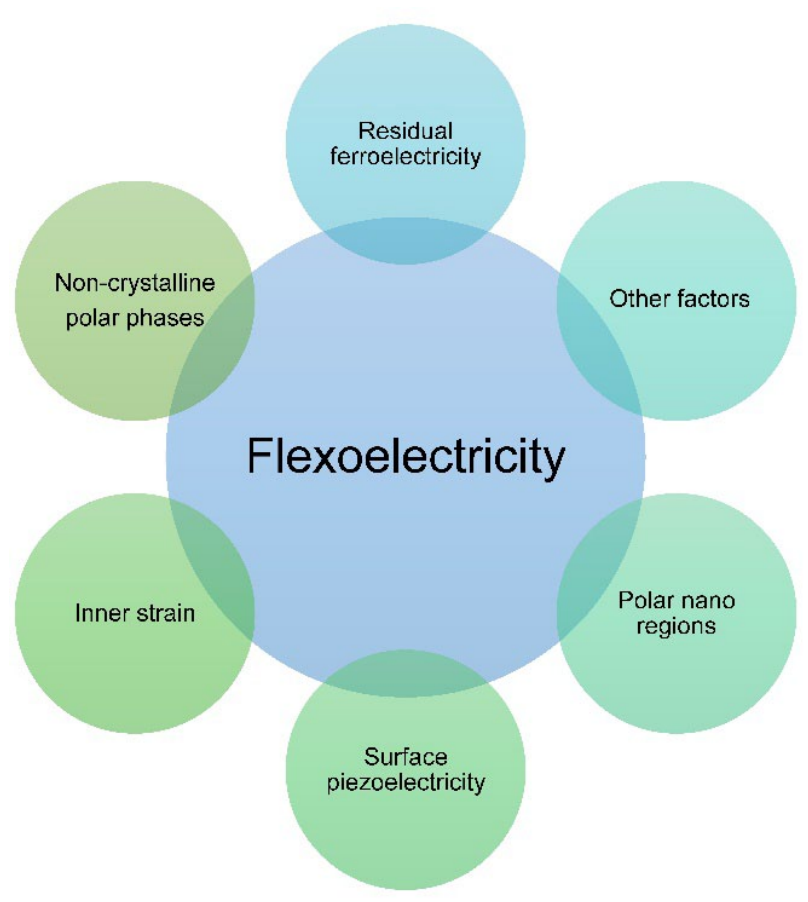

Fig. 3 Possible mechanisms of enhanced flexoelectricity in dielectrics. 
Table 1 Experimental and calculated values of flexoelectric coefficients for some materials

\begin{tabular}{|c|c|c|c|c|c|c|}
\hline \multirow{2}{*}{ Material } & \multicolumn{2}{|c|}{$\mu_{1111}(\mathrm{nC} / \mathrm{m})$} & \multicolumn{2}{|c|}{$\mu_{1122}(\mathrm{nC} / \mathrm{m})$} & \multicolumn{2}{|c|}{$\mu_{1212}(\mathrm{nC} / \mathrm{m})$} \\
\hline & Experiment & DFT & Experiment & DFT & Experiment & DFT \\
\hline GaAs & - & +0.51 & - & +0.85 & - & -0.84 \\
\hline $\mathrm{GaP}$ & - & +0.47 & - & +0.31 & - & -0.34 \\
\hline $\mathrm{ZnS}$ & - & -0.31 & - & -1.5 & - & -0.61 \\
\hline $\mathrm{KCl}$ & - & +0.40 & - & -0.12 & - & -0.23 \\
\hline $\mathrm{NaCl}$ & - & +0.41 & - & -0.12 & - & -0.21 \\
\hline $\mathrm{TiO}_{2}$ & - & - & 2 & - & - & - \\
\hline Polyvinylidene fluoride & - & - & 10 & - & - & - \\
\hline Epoxy resin & - & - & 30 & - & - & - \\
\hline Polyethylene & - & - & 6 & - & - & - \\
\hline Polyethylene terephthalate & - & - & 10 & - & - & - \\
\hline $\mathrm{SrTiO}_{3}$ & 0.2 & -0.89 & 7 & +2.3 & 5.8 & -6.6 \\
\hline $\mathrm{PbZrO}_{3}$ & - & - & 2.5 & - & - & - \\
\hline hydroxyapatite & - & - & 1.2 & - & - & - \\
\hline$(\mathrm{KNaLi})(\mathrm{NbSb}) \mathrm{O}_{3}$ & - & - & $2 \times 10^{3}$ & - & - & - \\
\hline BT-BZT & - & - & $2.5 \times 10^{4}$ & - & - & - \\
\hline $\mathrm{Pb}(\mathrm{ZrTi}) \mathrm{O}_{3}$ & - & - & $1 \times 10^{3}$ & - & - & - \\
\hline $\mathrm{Pb}_{0.3} \mathrm{Sr}_{0.7} \mathrm{TiO}_{3}$ & - & - & $2 \times 10^{4}$ & - & - & - \\
\hline PMN-PT & - & - & $(2-5) \times 10^{4}$ & - & - & - \\
\hline $\mathrm{PbMg}_{1 / 3} \mathrm{Nb}_{2 / 3} \mathrm{O}_{3}$ & $(3-4) \times 10^{3}$ & - & - & - & - & - \\
\hline PIN-PMN-PT & - & - & $1.1 \times 10^{5}$ & - & - & - \\
\hline $\mathrm{BaTiO}_{3}$ & $5 \times 10^{4}$ & -0.36 & $5 \times 10^{4}$ & +1.6 & - & -1.5 \\
\hline$\left(\mathrm{Ba}_{1-x} \mathrm{Sr}_{x}\right) \mathrm{TiO}_{3}$ & $1.15 \times 10^{5}$ & 10-20 & $1 \times 10^{5}$ & $6-10$ & - & - \\
\hline $\mathrm{Ba}\left(\mathrm{Ti}_{1-x} \mathrm{Sn}_{x}\right) \mathrm{O}_{3}$ & - & - & $4.5 \times 10^{4}$ & - & - & - \\
\hline
\end{tabular}

flexoelectric electric field will exist in the vicinity of the cracks of the hydroxyapatite.

The bio-membrane flexoelectricity was recently studied by using the droplet interface bilayer technology (DIB) [62-65]. The principle of DIB in this scenario, as shown in Fig. 4(c) [66], is by using lipids as the organic surfactant in oil-water emulsion, in order to create biomimetic membranes at the intersection of neighboring droplets. When two droplets are brought into contact, the lipid monolayers adhere together which act as a capacitor. The contact angle at the monolayer-bilayer meniscus is determined by the balance of the tension between the two interfaces. Intriguingly, this technique can be utilized to explore the mechano-transduction and interfacial properties of unsupported liquid biomimetic membranes. Freeman et al. [66] reported that high-frequency membrane deformation is capable of producing a significant flexoelectric current, whose value is related to the curvature of the interfacial membrane, as shown in Fig. 4(d). This result suggested that flexoelectricity in bio-membrane can drive the activation of tension-gated channels within the membrane $[67,68]$.

\section{4 Flexoelectricity in liquid crystals}

The liquid crystal flexoelectricity stems from the 1970s and has been regarded as one of the fundamental properties of this kind of materials. In general, the flexoelectric coefficients in liquid crystals are mainly represented by two independent components, the component $e_{1}$ and the component $e_{3}$, which respond to the splay deformation and bend deformation, respectively. Up till now, two independent ways have been developed to measure the flexoelectric components $e_{1}$ and $e_{3}$ in liquid crystals. One is reported by Trabi et al. [69] based on the converse flexoelectric effect. As shown in Fig. 5(a), to yield a non-uniform electric field, interdigitated electrodes were deposited onto the liquid crystal surface. Due to the coupling of converse flexoelectricity, the liquid crystal was deformed into a 
(a)

(c)

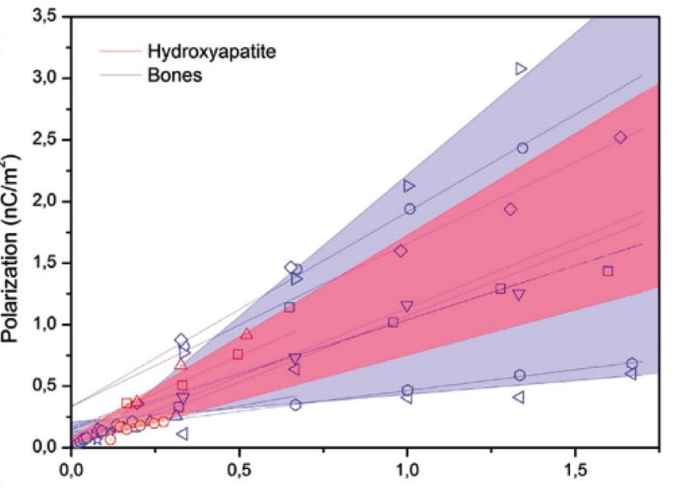

Strain gradient $\left(\mathrm{m}^{-1}\right)$ (b)

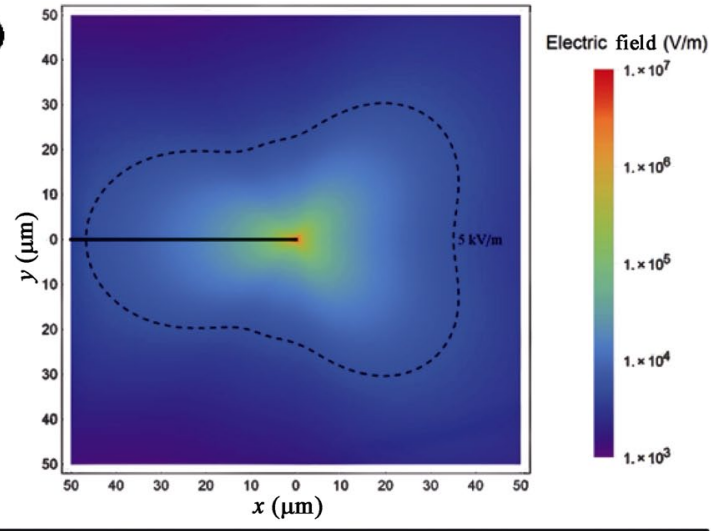

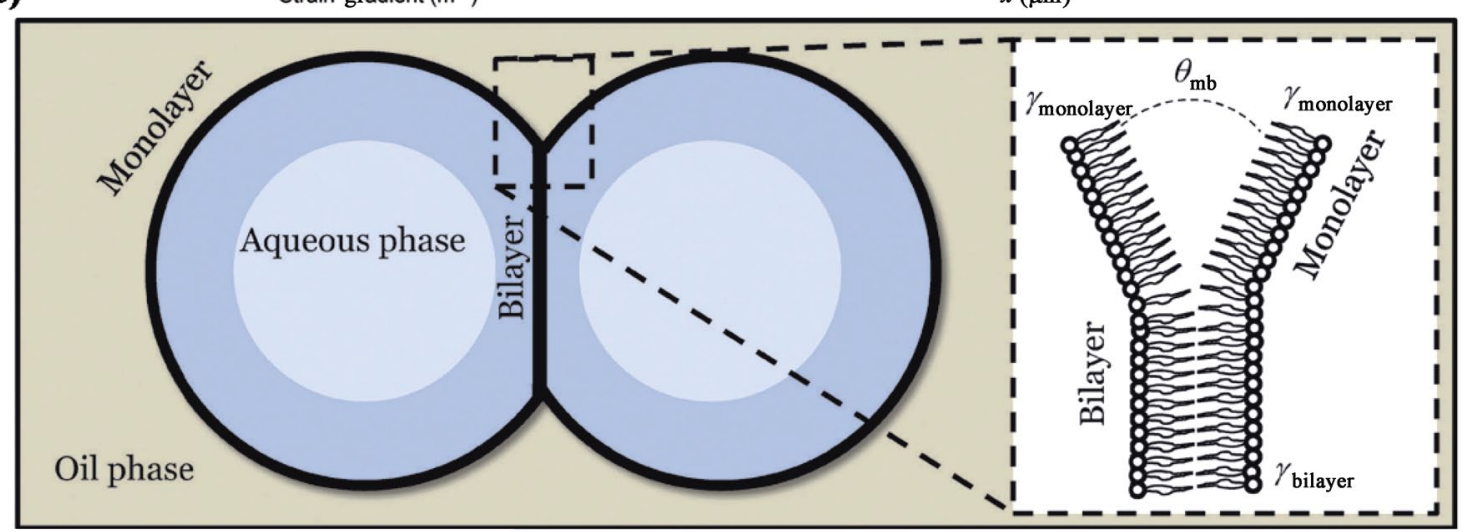

(d)
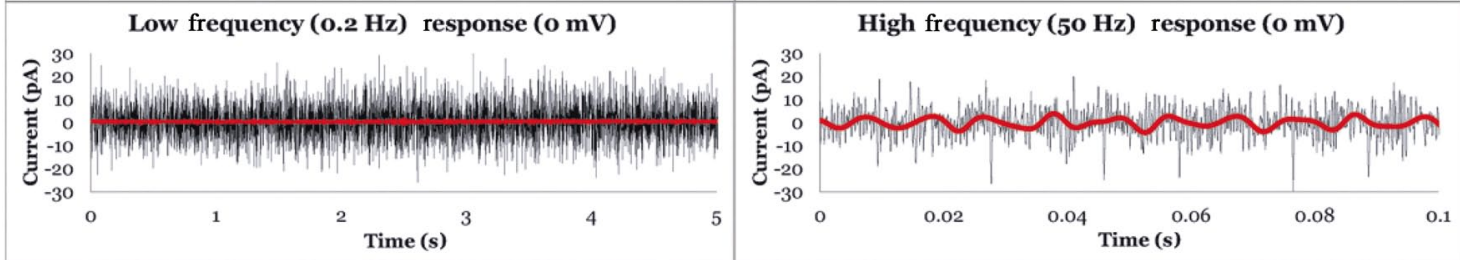

Fig. 4 (a) The flexoelectric coefficient is the constant of proportionality between strain gradient (bending) and bendinginduced polarization. (b) Calculated flexoelectric field distribution around a microcrack in bone mineral, the flexoelectric field is biggest at the crack tip and decays progressively away. Reproduced with permission from Ref. [61], C WILEY-VCH Verlag $\mathrm{GmbH} \&$ Co. KGaA, Weinheim 2018. (c) The Droplet-Interface Bilayer (DIB) technique. This technique uses lipids as an organic surfactant in an oil-water emulsion, creating biomimetic membranes at the intersection of neighboring droplets. When the droplets are brought into contact, the lipid monolayers adhere together in a lipid bilayer, often approximated as a capacitor. The contact angle at the monolayer-bilayer meniscus is determined by the balance of the tension between the two interfaces. (d) At high frequencies of oscillation $(50 \mathrm{~Hz})$, which shifts the membrane deformation mechanic from gradual growth and reduction to elastic strain and bending, then producing flexoelectric current. Reproduced with permission from Ref. [66], (C) Royal Society of Chemistry 2016.

splay structure, and this deformation could be quantified by the distortion of the birefringence pattern. The other method is proposed by Harden et al. [70] through the direct flexoelectric effect, as shown in Fig. 5(b). This method is similar to the measurement of flexoelectricity in dielectrics. The studied nematic liquid crystal (NLC) cells were firstly fixed in a flexible container, and then bent by using a loudspeaker. Later, the NLC was oscillated between two fixed posts, and a large flexoelectric current could be induced. For most of the liquid crystals, the typical value of flexoelectric components $e_{1}$ and $e_{3}$ is $1-100 \mathrm{nC} / \mathrm{m}$ [71]. Compared with the ferroelectrics, the flexoelectric coefficients of liquid crystals are relatively small. However, regardless its small coefficients, the flexoelectricity often plays a dominant role in liquid crystals, especially in the widely developed liquid crystal display technology [72-78]. Recently, Lee et al. [79] found that the highly distortion of the liquid crystal display could be quantitatively measured by the variation of the flexoelectric polarization, 
(a)

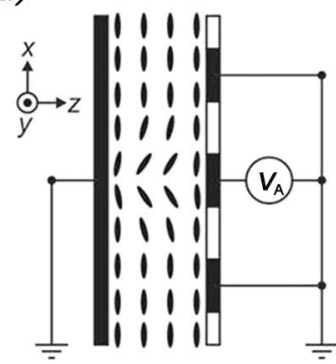

(c)

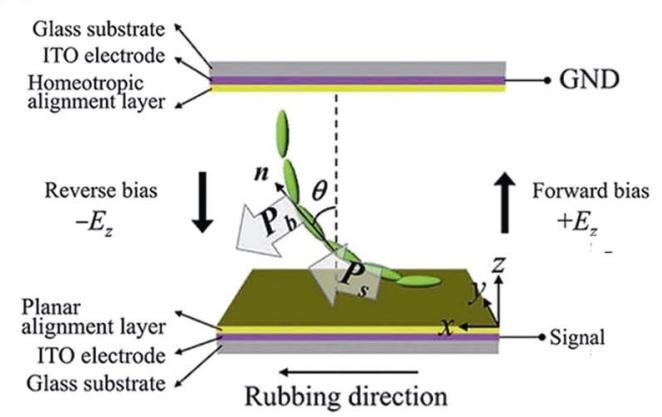

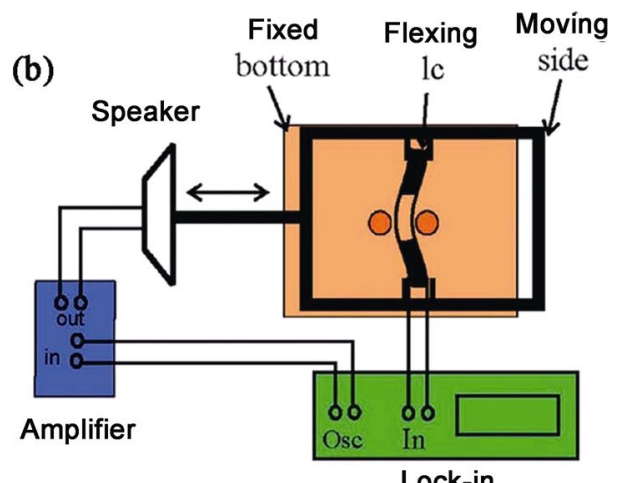

Lock-in

(d)

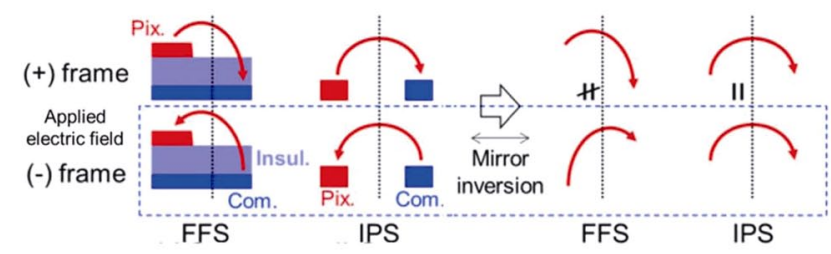

Fig. 5 (a) Schematic view of indirect measurement of flexoelectric coefficient in liquid crystal. An electric field was applied onto the interdigitated electrodes and the flexoelectric coefficients was extracted by the distortion of birefringence images. Reproduced with permission from Ref. [69], (C) AIP Publishing 2008. (b) Schematic view of the direct measurement of flexoelectric coefficient in liquid crystal. Reproduced with permission from Ref. [70], (C) American Physical Society 2006. (c) Illustration of the direction of the electric field and the flexoelectric polarization of liquid crystal under the splay and bend deformations. Reproduced with permission from Ref. [79], (C) Taylor \& Francis 2017. (d) Illustration of the field symmetry for positive and negative frames, where the IPS refers to in-plane-switching, FFS refers to fringe-field-switching. Reproduced with permission from Ref. [80], (c) Optical Society of America 2016.

as shown in Fig. 5(c). Moreover, the image flicker problem of liquid crystal mixtures which shows negative dielectric anisotropy can be minimized by the flexoelectric effect [80,81] (as shown in Fig. 5(d)).

\section{5 Flexoelectricity in semiconductor}

Generally speaking, the electro-mechanical coupling is only limited to dielectrics due to the requirement of insulation. But more and more studies have disclosed that semiconductors can be regarded as electromechanical materials, e.g., the $\mathrm{ZnO}$ semiconductor is a piezoelectric material [82-84]. In 2016, Narvaez et al. [85] made a new breakthrough in flexoelectricity and greatly broadened the concept of flexoelectricity into semiconductors. A dead layer mechanism was proposed and the measured flexoelectricity in some semiconductors can reach a level to $1000 \mu \mathrm{C} / \mathrm{m}$.

As shown in Fig. 6(a), for an arbitrary bulk insulator such as $\mathrm{BaTiO}_{3}$ single crystal, the surface piezoelectricity exists due to the symmetry breaking in boundary. At the top and bottom surfaces, the piezoelectric polarization is usually orientated in opposite direction so that the macro piezoelectricity is zero. Once bending this bulk material, a net polarization exists because the bending induced strain direction of the top and bottom surface is opposite. That is why the surface piezoelectricity can couple to the enhanced flexoelectricity in the bulk insulators [86]. Similarly, this mechanism also works in semiconductor owing to the existence of dead layer. The dead layer, typically in several micrometers thick, could accumulate the top and bottom surface piezoelectricity, and results in a significant net polarization when the semiconductor is bent. As shown in Fig. 6(b), the oxygen reduced $\mathrm{BaTiO}_{3}$ single crystal which acts as a good ionic conductor, shows an extremely enhanced flexoelectric-like response. This phenomenon was also found in Nb-doped $\mathrm{TiO}_{2}$ semiconductor. Interestingly, the flexoelectricity in semiconductor shows a linearized thickness dependence. The measured effective flexoelectric coefficients were directly proportional with the material thickness, as shown in Fig. 6(c). 
(a)

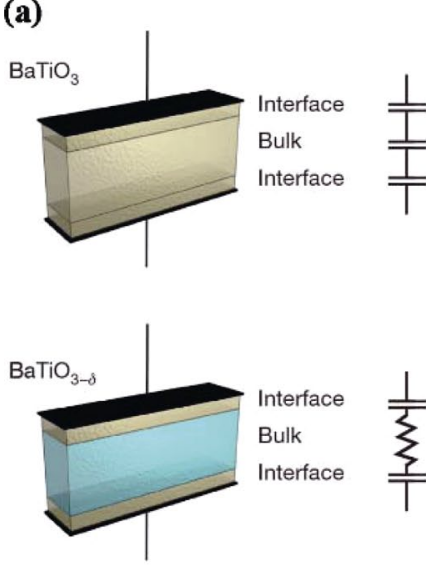

(b)

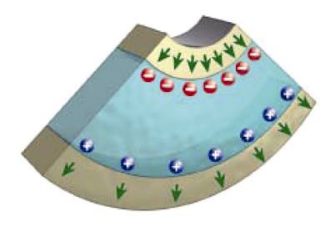

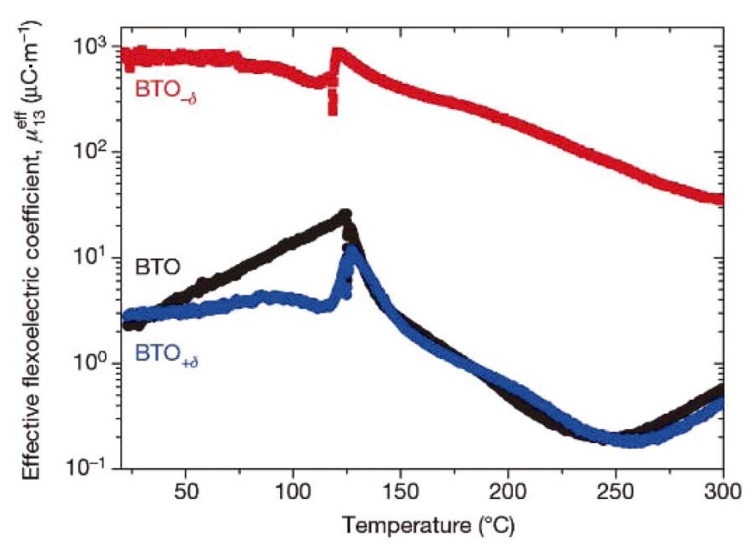

(c)

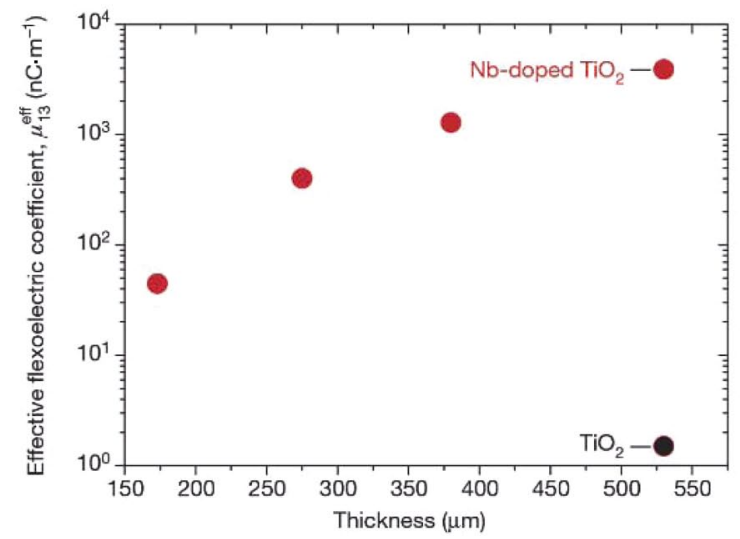

Fig. 6 (a) Schematic view of the barrier layer mechanism. For both the bulk insulators and the semiconductors, the surface piezoelectricity will contribute to the flexoelectric coefficients. The barrier layer in semiconductor will block the free charge and have a giant contribution for flexoelectric coefficients. (b) Temperature dependence of the effective transverse flexoelectric coefficients in pure bulk $\mathrm{BaTiO}_{3}$ single crystal, reduced $\mathrm{BaTiO}_{3}$ single crystal, and oxidized $\mathrm{BaTiO}_{3}$ single crystal. (c) Thickness dependence of the effective transverse flexoelectric coefficients in $\mathrm{Nb}$-doped $\mathrm{TiO}_{2}$ semiconductor. Reproduced with permission from Ref. [85], (C) Springer Nature 2016.

Inspired by this attractive result, one can expect that with proper design, the application of semiconductor flexoelectricity will play an important role in the optimization of the integrated circuit and electromechanical semiconductor devices.

\section{Theoretical calculation of flexoelectricity}

During the past few years, the studies of flexoelectricity were not solely focused on the experiment level, but also led to a lot of theoretical progress. It is worth noting that the size effect of flexoelectricity was firstly proposed by theoretical prediction rather than any experiment [14]. Numerous theoretical studies have demonstrated that the strain gradient becomes large in nanoscale, at which the flexoelectricity may even be competitive with piezoelectricity [87-93]. Specifically, in epitaxial ferroelectric thin films [94], the simulated strain gradient can reach up to $10^{6}-10^{7} \mathrm{~m}^{-1}$, which may make the flexoelectric polarization higher than the piezoelectric one.

The general theory of flexoelectricity was recently developed. Many of them were focused on the origin of the enhancement of flexoelectricity in some typical materials [95-97]. Theoretical work on flexoelectricity dates back to the papers by Mashkevich and Tolpygo [98], who first proposed the effect, and Kogan [8], who formulated the first phenomenological theory. According to the theory proposed by Yudin et al. [99], the flexoelectric polarization can be accounted for four parts, i.e., surface piezoelectricity, bulk piezoelectricity, surface flexoelectricity, and bulk flexoelectricity [20]. All these factors contribute almost equally to the flexoelectric coefficient. However, a different viewpoint was stated hereupon by Resta [52] who built the polarization theory 
based on the elemental cubic crystal. His theoretical result manifested that the intrinsic flexoelectricity was a purely bulk effect, where the surface contribution was totally excluded. Later, Yurkov and Tagantsev. [100] argued that the direct bulk flexoelectric response of a finite sample essentially depends on the surface polarization energy. Recently, Zhang et al. [54] calculated that the surface piezoelectricity which widely exists in all the unpolarized ceramics and single crystals, is possibly dominant in the observed flexoelectricity. This statement also well matches their experimental results [54]. The controversy for this issue still exists and a comprehensive understood is urgently needed.

The first-principles calculation of flexoelectric coefficients was firstly developed in several semiconductors by Maranganti and Sharma [21] until 2009. Hong et al. $[22,23,101]$ demonstrated the first ab initio calculation of the longitudinal flexoelectricity for $\mathrm{BaTiO}_{3}$ and $\mathrm{SrTiO}_{3}$ by using a direct approach, where the strain gradient is realized by setting the lattice parameter of each atom. Meanwhile, they also developed an indirect way for calculating flexoelectric coefficients which was formulated in such a way that the tensor elements can be computed directly in the context of density-functional calculations, including electronic and lattice contributions [23]. To date, the first-principles method could be utilized into the calculation of other flexoelectric tensor component and much more materials, which is summarized in Table 1 [102-106].

An interesting theoretical progress of flexoelectricity we addressed is the application of topology optimization. This method, firstly proposed by Bendsøe and Kikuchi [107] in 1988, has been widely applied to optimize the material property [108,109], especially for the design of domains [110,111]. More recently, Ghasemi et al. [112,113] presented a new-type topology optimization method, which is based on a combination of isogeometric analysis (IGA), level set, and point wise density mapping techniques as shown (a)

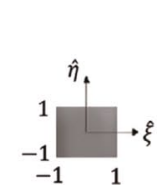

Parent element

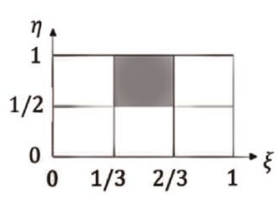

Parameter space

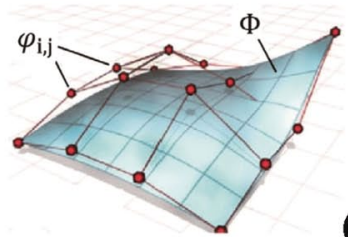

Physical space
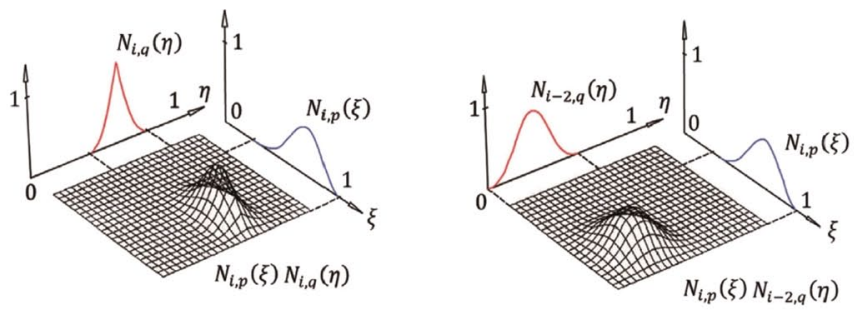

Typical shape functions of order $p$ and $q$ (b)

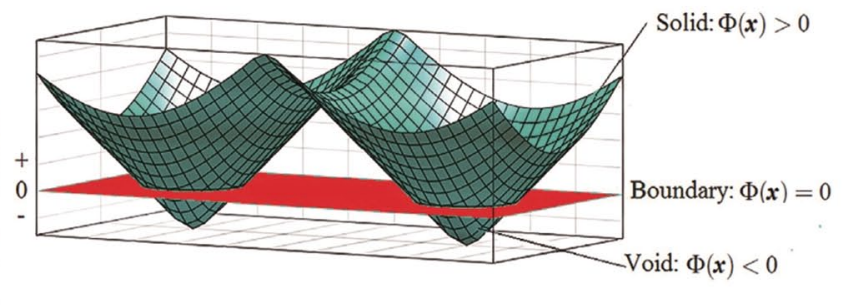

(c)
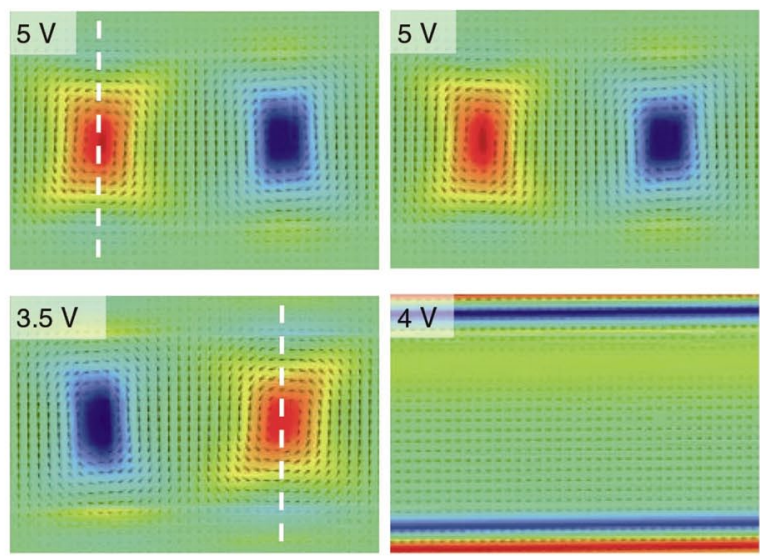

$4 \mathrm{~V}$

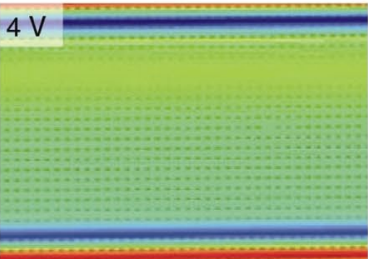

Fig. 7 (a) IGA concept. Each element in the physical space is the image of a corresponding element in the parameter space, and the parameter space is discretized by knot vectors. Control points in IGA are used to discretize the geometry and define the degrees of freedom. Reproduced with permission from Ref. [112], (C) Elsevier B.V. 2016. (b) The level set function which easily copes with the various numbers of phases, is efficiently satisfied with the multiple constraints. This function intrinsically avoids overlap or vacuum among different phases. Reproduced with permission from Ref. [113], (c) Elsevier B.V. 2017. (c) Simulated vortex patterns represented by the vorticity calculated from the polarization vector field. A vortex/anti-vortex pair region marked by the dashed line is chosen to illustrate the flexocoupling effects in what follows, and the double-arrow symbol indicates an anti-phase boundary. Typical vortex pair pattern under different flexocoupling of the STO layers. Reproduced with permission from Ref. [114], (C) The Author(s) 2017. 
in Fig. 7(a) and Fig. 7(b), respectively. Through this topology optimization, significant enhanced flexoelectric coefficients can be obtained in designated materials.

Other theoretical calculation methods such as finite element method, phase-filed modeling, and density functional theory, are also developed to explore the direct and converse flexoelectric effect [114-124]. It is worth noting that $\mathrm{Li}$ et al. [114] reported the application of flexoelectric phase-field model [125] in calculating the polar vortices of $\mathrm{PbTiO}_{3} / \mathrm{SrTiO}_{3}$ super-lattices. The results suggested that the induced polarization (represented by the vortex/anti-vortex pair region) is highly related to the given flexoelectric coefficients, as shown in Fig. 7(c).

\section{Applications of flexoelectricity}

Here we will emphasize on the recent developed applications of flexoelectricity in several fields, including flexoelectric energy harvester, flexoelectric sensors and actuators, domain engineering, liquid crystal displaying, and some other open applications, e.g., flexoelectricity-tuned photovoltaic effect. In the end, we also list some possible applications where the flexoelectricity might be involved.

\section{1 Energy harvesters}

Energy harvesters are those devices which can collect energy from the external sources like wind energy, solar power, thermal energy, etc. Specifically, the electromechanical energy harvester can collect energy from the mechanical vibrations [126-132]. Recently, the newly designed energy harvester which collect energy from the fluctuation of the vibrations (strain gradient) was proposed. Owing to its special sizedependent effect, the flexoelectric energy harvester is suitable to be integrated into small size, where a huge strain gradient can be generated. Till now, with the proper design, the mechanical-electrical energy converting efficiency of the flexoelectric energy harvester can reach to $6.6 \%$ [133-135].

The potential of flexoelectricity as energy harvester has been predicted by many works. Wang and Wang [136] developed an analytical model for vibrationbased circular energy harvester that consists of a flexoelectric layer and a substrate layer. The result indicated that both the power output (as shown in Fig. 8(a)) and the energy conversion efficiency (as shown in Fig. 8(b)) of the flexoelectric energy harvester are much larger than that of the classical piezoelectric energy harvesters. Particularly, when the thickness $\left(h_{\mathrm{p}}\right)$ of the flexoelectric layer is reduced to less than $100 \mathrm{~nm}$, the power output of the flexoelectric energy harvester is almost 12 times larger than that of the piezoelectric energy harvester [137]. Choi and Kim [138] fabricated a flexoelectric energy harvester device which collects energy by a PVDF thin film deposited on the cantilever substrate. The photograph of this device is shown in Fig. 8(c). Han et al. [139] prepared a flexoelectric nanogenerator which is consisted of direct-grown piezoelectric PZT on multi-walled carbon nanotubes. This device can repeatedly generate a voltage output of $8.6 \mathrm{~V}$ and a current output of $47 \mathrm{nA}$ at a mechanical force of $20 \mathrm{~N}$, which is promising for harvesting the mechanical energy. Moreover, Zhu et al. [140] designed a flexible flexoelectric fiber which is made of curved piezoelectric composites. As shown in Fig. 8(d), these fibers possess a stable upward self-poling which is induced by flexoelectricity, exhibited a large electric output comparable to those of the piezoelectric nanogenerators.

\section{2 Actuators}

Piezoelectricity has been widely used for sensors and actuators. Similarly, the flexoelectricity could also be utilized for sensing and actuating applications, especially for nano-sized devices. Compared with the piezoelectric sensors and actuators, the flexoelectric ones are not limited by the crystal symmetry of the materials and the working temperature $[141,142]$. The past few years have witnessed many progresses in this aspect. As shown in Fig. 9(a), Zhang et al. [143] designed a curved flexoelectric actuator by using non-polarized PVDF. This actuator exhibited a good actuating property with a resolution of the displacement reaching up to $1.0 \mathrm{~nm}$ and a largest displacement as high as $63.6 \mathrm{~nm}$ (as shown in Fig. 9(b)). It is worth highlighting that Bhaskar et al. [144] implemented a flexoelectric actuator made of micron-sized barium titanate (as shown in Fig. 9(c)) which is fully compatible for the semiconductor silicon technology. The performance result (Fig. 9(d)) suggests that this flexoelectric actuator displays comparable performance to the actuators use lead-containing piezoelectric material. 


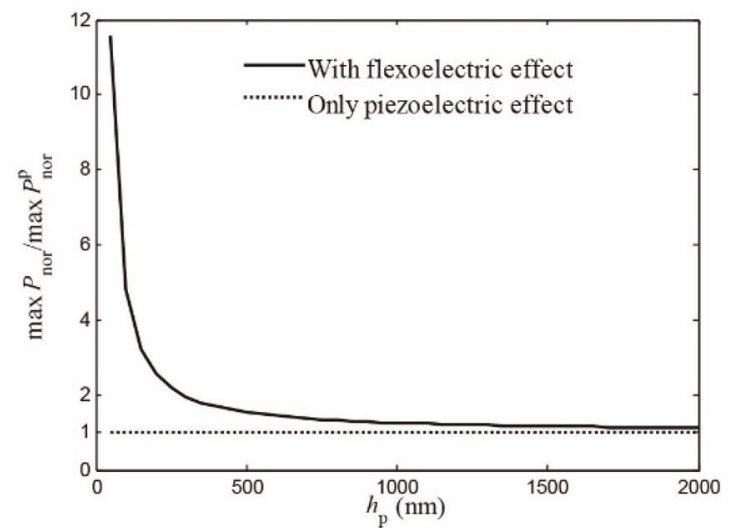

(a)

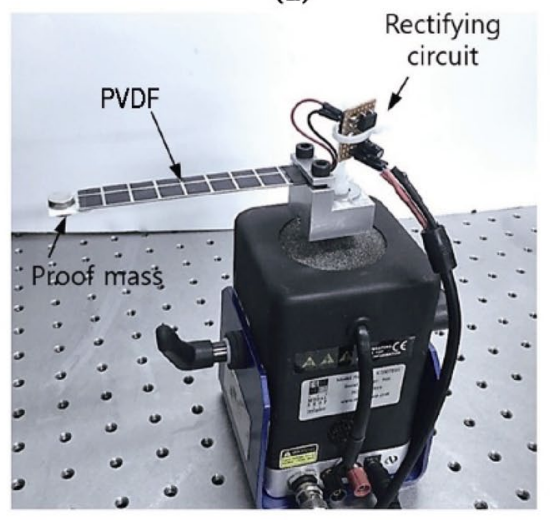

(c)

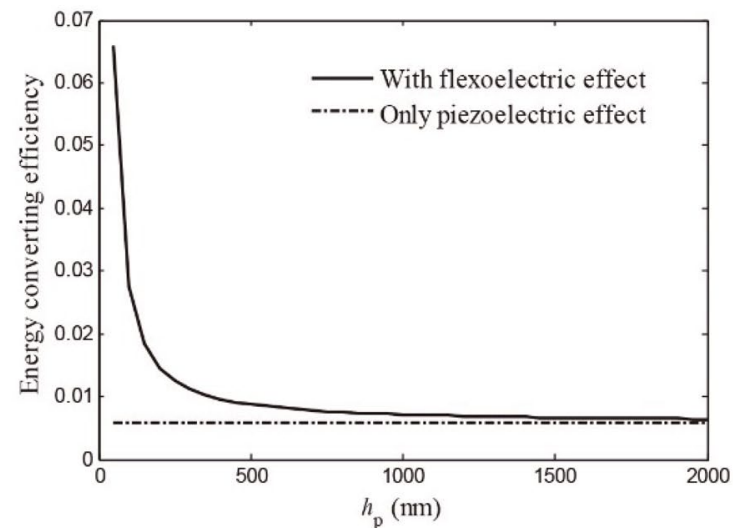

(b)

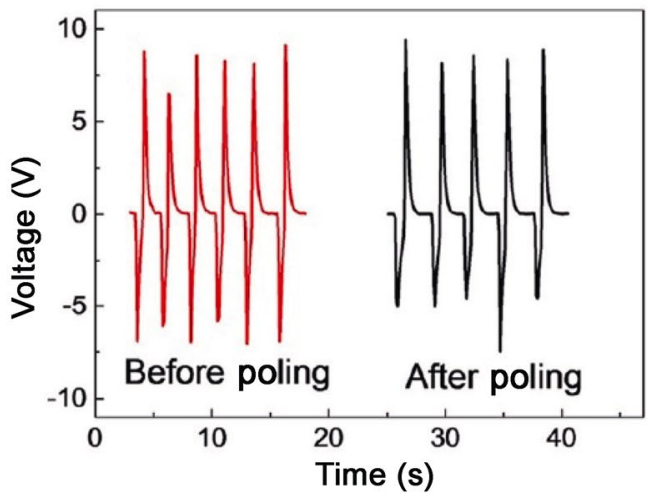

(d)

Fig. 8 (a) The maximum normalized power output of vibration-based circular energy harvester as a function of the thickness of the flexoelectric layer. (b) The energy converting efficiency of vibration-based circular energy harvester as a function of the thickness of the flexoelectric layer. Reproduced with permission from Ref. [136], (C) Elsevier Ltd. 2018. (c) The photography of the PVDF-based energy harvester which could collect energy from the excited vibrations. Reproduced with permission from Ref. [138], (C) IOP Publishing Ltd. 2017. (d) Comparison of the transient output voltage between the unpolarized nanogenerator and polarized nanogenerator. Reproduced with permission from Ref. [140], C Elsevier Ltd. 2018.

\section{3 Sensors}

The flexoelectric sensors are becoming increasingly popular because of their small size, absence of depoling and aging problem, and lead-free composition [145]. The state-of-the-art flexoelectric sensor has been successfully utilized in many fields such as structural health monitoring, crack detector, and curvature detector [146,147]. Recently, Yan et al. [148] designed a BST-based flexoelectric curvature sensor, which can transfer the bending deflections directly to the charge output. The working principle of this sensor is illustrated in Fig. 10(a). To precisely detect the bending deflection, two BST micro curvature sensors were attached onto the center side surfaces of an aluminum beam, located symmetrically with respect to its neutral axis. The relationship between flexoelectric charge outputs of the BST sensors and the curvature (strain gradient) is shown in Fig. 10(b). The sensitivity of this curvature sensor, defined by the slopes of the charge outputs versus curvature, can reach up to $1 \mathrm{pC} / \mathrm{m}$. In addition, Merupo et al. [149] investigated the potential use of $6.6 \mu \mathrm{m}$-thick soft polyurethane films as the large curvature sensor by subjecting flexible aluminum foil/PET bi-layered substrate to large deflections. A curvature of about $80 \mathrm{~m}^{-1}$ corresponding to a radius of curvature of $\sim 1.2 \mathrm{~cm}$ could be effectively sensed under a low frequency $(3 \mathrm{~Hz}$ ) bending motion. The pseudosinusoidal time dependence of the output charge of this sensor is shown in Fig. 10(c). Kwon et al. [150] reported recently that the flexoelectricity can work in the microphone. The designed sensitivity of the fabricated barium strontium titanate $\left(\mathrm{Ba}_{0.65} \mathrm{Sr}_{0.35} \mathrm{TiO}_{3}\right)$ microphone was very high and almost frequencydependent in wide frequency range, as shown in Fig. 10 (d). 
(a)

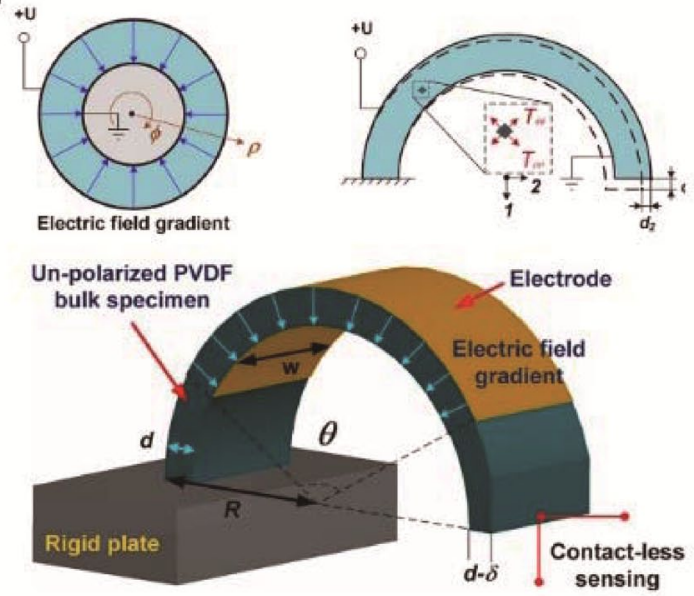

(c)

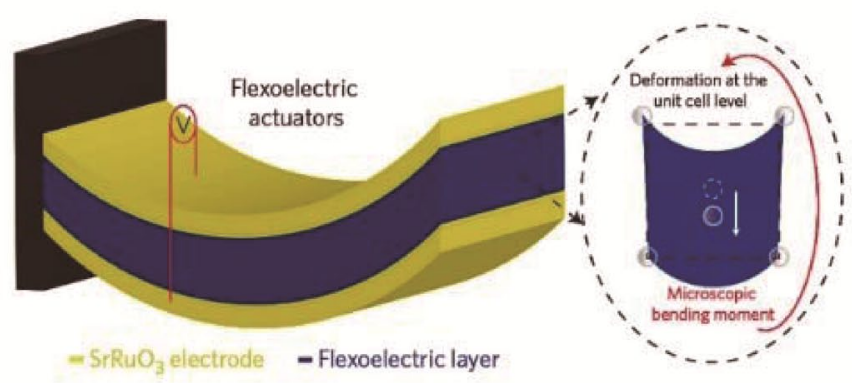

(b)

(d)
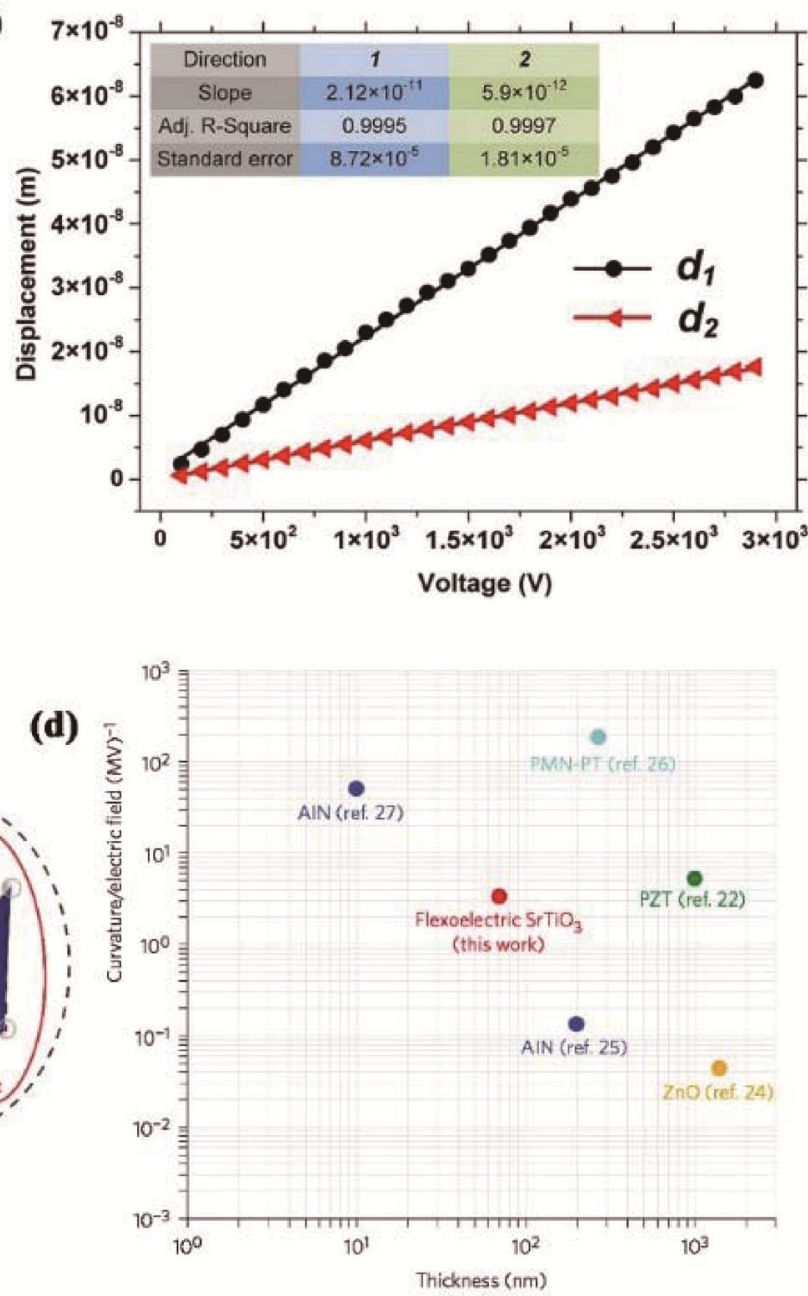

Fig. 9 (a) Schematic diagram of the PVDF-based actuator. The symbol $\theta$ represents the angle of electrode attachment. To achieve a uniform electric field gradient and application safety, $\theta$ in this actuator is set as $120^{\circ}$. (b) Induced displacement as a function of voltage in PVDF-based actuator. Reproduced with permission from Ref. [143], (C) AIP Publishing 2017. (c) Schematic view of the $\mathrm{SrTiO}_{3}$-based actuator. Particularly, the flexoelectric layer is compatible with silicon or any of its gate dielectrics in a completely complementary metal oxide semiconductor-compatible environment. (d) Actuating performance comparison of the $\mathrm{SrTiO}_{3}$-based actuator with other actuators. Reproduced with permission from Ref. [144], C Springer Nature 2015.

\section{4 Domain tailoring and polarization switching}

Another attractive application of flexoelectricity should be addressed is the domain tailoring and polarization switching. As we may know that the ferroelectric materials are characterized by their spontaneous polarization, which can be switched by applying an external electric field. As suggested by $\mathrm{Lu}$ et al. [1] and Catalan et al. [2], in a nano-sized material, e.g., ultrathin ferroelectric film, the spontaneous polarization can be switched by mechanical strain gradient. This powerful function has attracted increasing interests and hence makes heterostructure thin film a natural scenario for the flexoelectric applications.
Recently, Gómez et al. [151] prepared an epitaxial growth of columnar porous $\mathrm{BaTiO}_{3} / \mathrm{LaSrMnO}_{3} / \mathrm{SrTiO}_{3} /$ $\mathrm{Si}(001)$ heterostructures by using a complicated recipe which both combines the molecule beam epitaxy and polymer assisted deposition technology. The illustration of the preparation process is shown in Fig. 11(a). The results proved that the ferroelectric polarization of this heterostructure thin film can be reversed by a mechanical load in epitaxial columnar nanostructures, as shown in Fig. 11(b). Similar phenomenon was also found in $\mathrm{PbTiO}_{3}$ thin films [152]. It was experimentally proved that, as shown in Fig. 11(c), the mechanical force shows a comparable ability relative with the electrically method for switching the domain of the 
(a)

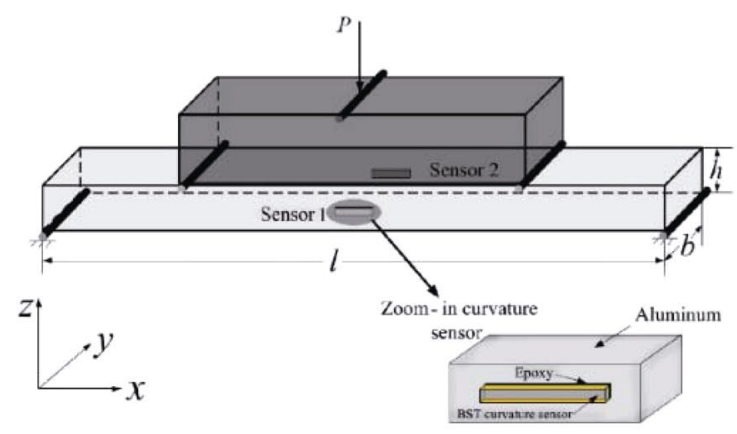

(c)

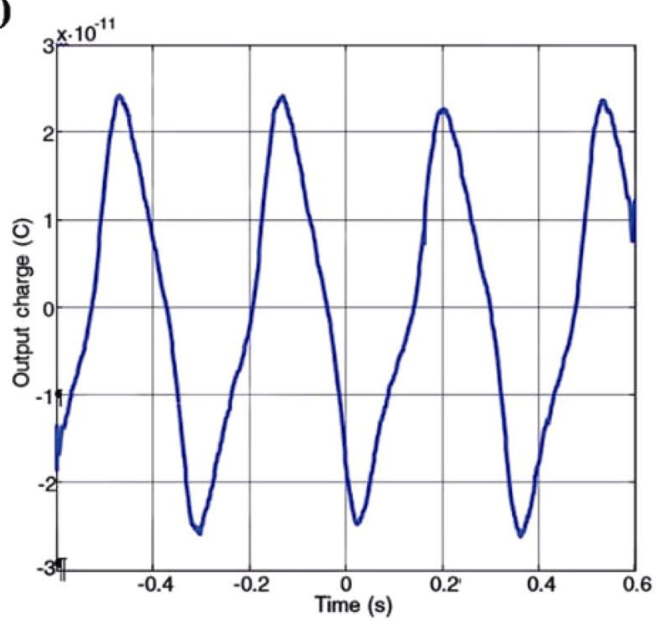

(b)

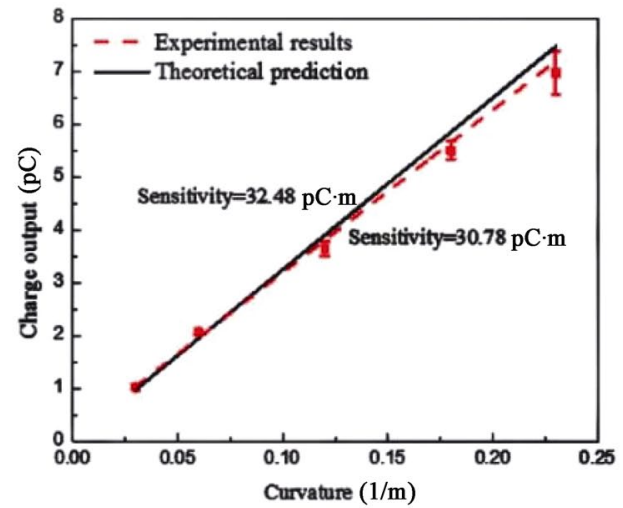

(d)

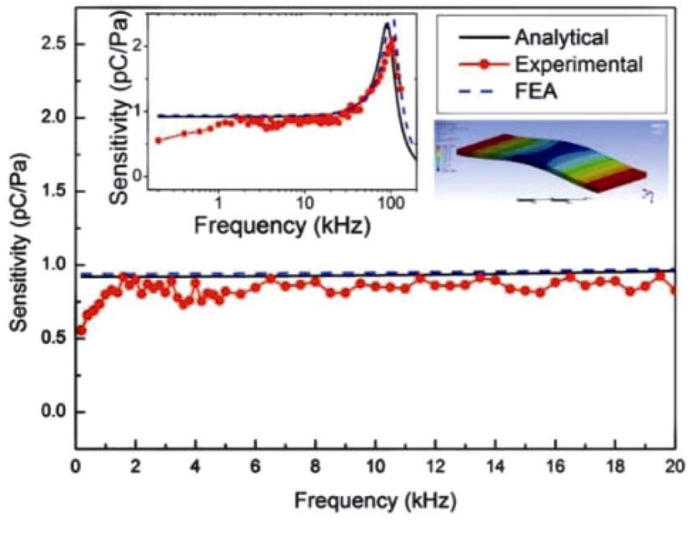

Fig. 10 (a) Beam curvature sensing: BST curvature sensor attached to beam. (b) Relationship between charge output and beam curvature-experimental results of BST curvature sensor. Reproduced with permission from Ref. [148], C SPIE 2013. (c) Real time dependence of the output electric charge of the $6.6 \mu \mathrm{m}$ thick curvature sensor. Reproduced with permission from Ref. [149], (C) AIP Publishing 2017. (d) The analytical, experimental sensitivity of the flexoelectric microphone at low frequency range (inset: overall sensitivity). Reproduced with permission from Ref. [150], C IOP Publishing Ltd. 2016.

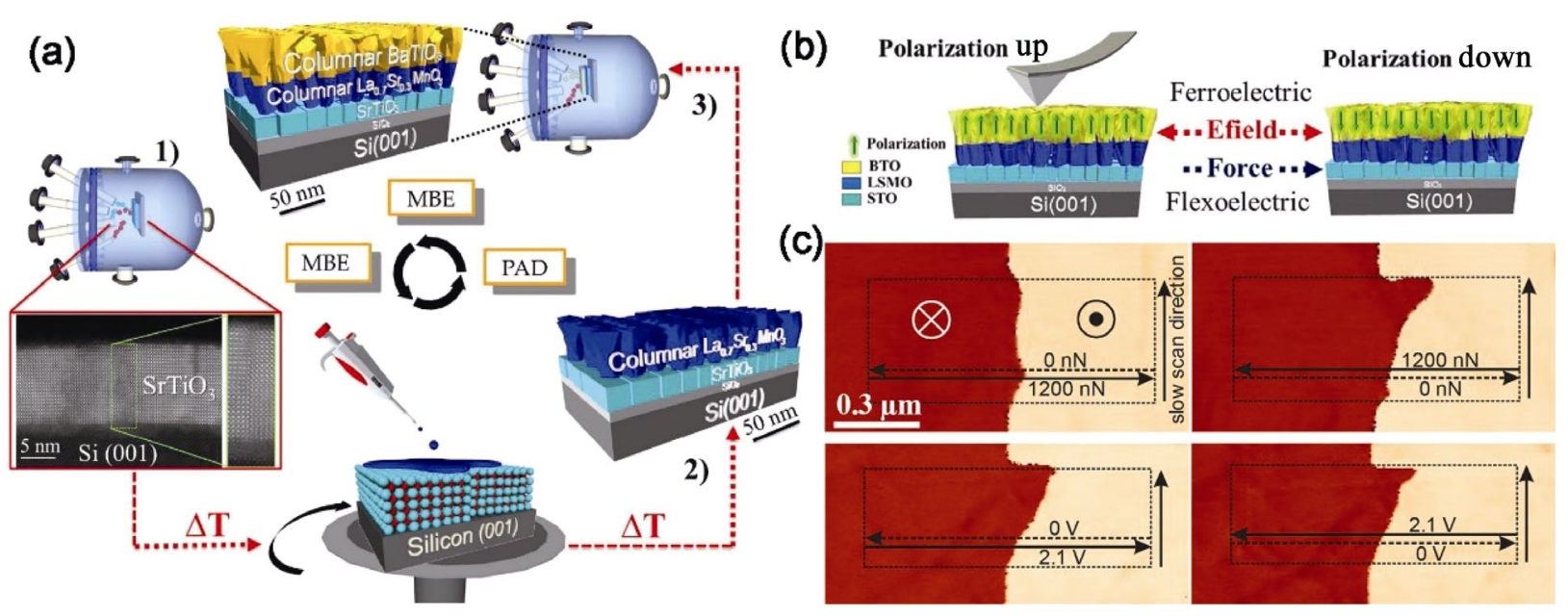

Fig. 11 (a) A new thin film preparation approach which combines molecule beam epitaxy and polymer assisted deposition. The complex epitaxial heterostructures were grown in three different stages. (b) Schematic view of the switching of the polarization by an electric field or by a mechanical force across the columnar BTO/LSM/STO/Si(001) heterostructure at room temperature. Reproduced with permission from Ref. [151], C WILEY-VCH Verlag GmbH \& Co. KGaA, Weinheim 2017. (c) Mechanical writing and electrical writing induced variation of the piezoresponse force microscopy phase images of the $\mathrm{PbTiO}_{3}$ film. The tip-induced pressure leads to the switching of the upward polarization $\left(\mathrm{c}^{+}\right.$domain) to the downward polarization ( $\mathrm{c}^{-}$domain). Reproduced with permission from Ref. [152], (C) AIP Publishing 2017. 
$\mathrm{PbTiO}_{3}$ thin films.

Moreover, with the help of flexoelectricity, the self-polarization direction of ferroelectric thin films, which is of difficulty in control in previous studies, is feasible to be changed by tuning the substrate interfaces and film thicknesses [153,154]. Park et al. [155] demonstrated that the multiple domain switching pathways in multiaxial ferroelectric materials can be selectively controlled by a newly realized trailing flexoelectric field, specifically, by the motion of a mechanically loaded scanning probe microscope tip. The illustration of this trailing flexoelectric field is shown in Fig. 12(a). Experimental results proved that the ferroelectric switching angle of multiferroic magnetoelectric $\mathrm{BiFeO}_{3}$ thin film can be stably selected at $71^{\circ}$ ferroelastic switching or $109^{\circ}$ ferroelectric switching. The in-plane polarization and out-of-plane polarization in response to the loading forces of the moving tip shows a significant orientation dependence, as shown in Fig. 12(b) and Fig. 12(c), respectively.

In addition, with the help of the flexoelectricity, the microscopic tip can also be used to explore dynamic ionic behavior [156] and manipulate the oxygen vacancies [157] (as shown in Fig. 12(d) and Fig. 12(f)). Obviously, the application of strain gradient into the ultra-thin film can result in different vacancy formation.

\section{5 Open applications}

Recent studies showed that the flexoelectricity can be linked to many other important physical behaviors, and hence bringing the flexoelectricity into the totally open applications. For example, the transverse shear surface acoustic waves have been found could propagate near the flat surfaces of all crystalline dielectrics because of the existence of flexoelectric effect [158-160]. Liu et al. [161] proposed that the bended thermoelectric $\mathrm{BiTe}_{3}$ film can present bulk photovoltaic effect at infrared wavelengths, which is possibly induced by the flexoelectric effect, as shown in Fig. 13(a). Yang et al. [162] proved that the application of flexoelectricity can cause the so-called flexo-photovoltaic effect. In their experiment, the large strain gradient (generated by the atomic force microscopy tip) and $405 \mathrm{~nm}$ laser (a)
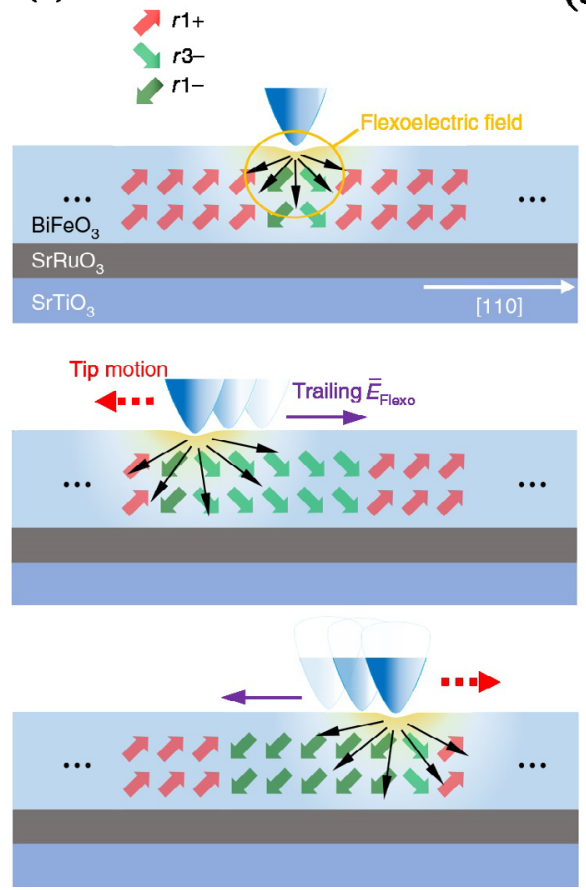

(b)

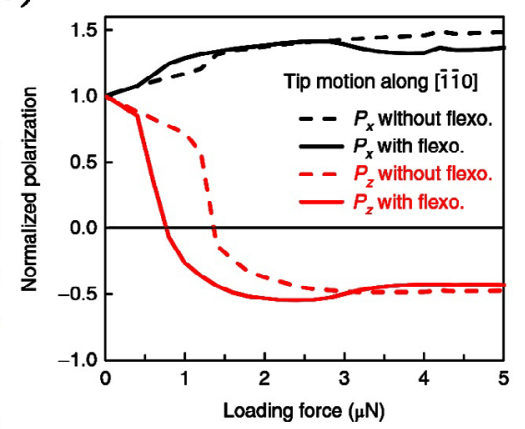

(d)

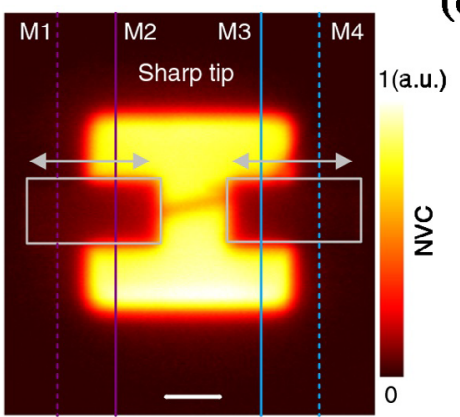

(c)

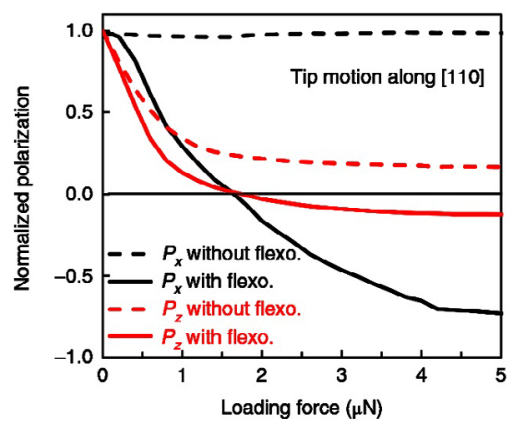

(e)

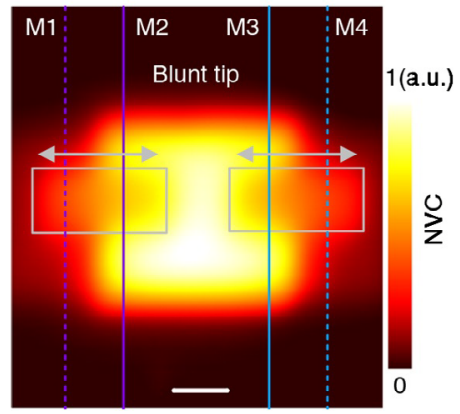

Fig. 12 (a) Schematic of polarization switching due to the trailing flexoelectric field tracing the SPM tip motion. The in-of-plane polarization $P_{x}$ (black) and out-of-plane polarization $P_{z}$ (red) as a function of loading forces with tip motion along [110] orientation (b) and [110] orientation (c). Reproduced with permission from Ref. [155], (C) Springer Nature 2018. (d) and (e) The normalized vacancy concentration maps after mechanical scanning, where (d) and (e) were performed using a sharp and blunt tip with a contact force of $9.5 \mu \mathrm{N}$, respectively. Reproduced with permission from Ref. [157], C) The Author(s) 2017. 


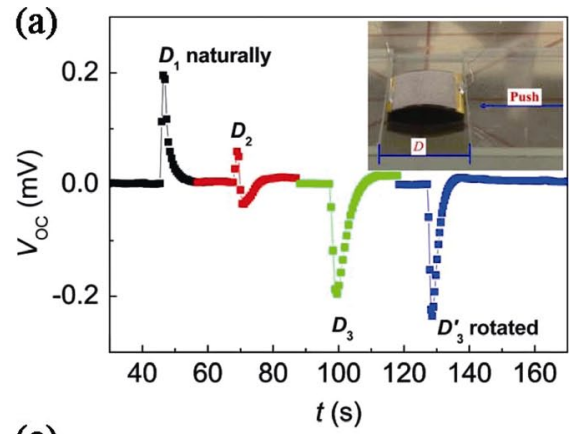

(c)

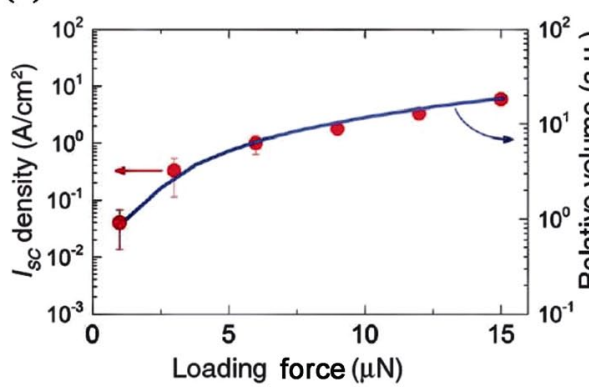

(e)
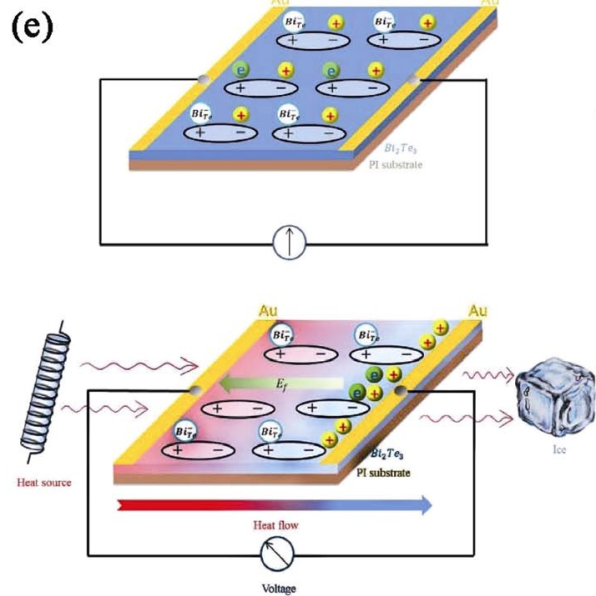

(b)

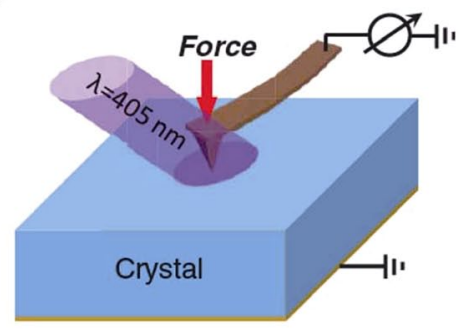

(d)

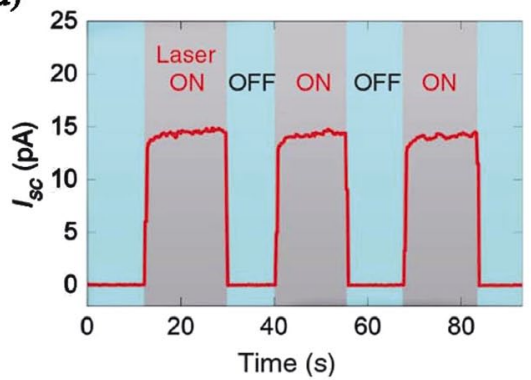

(f)

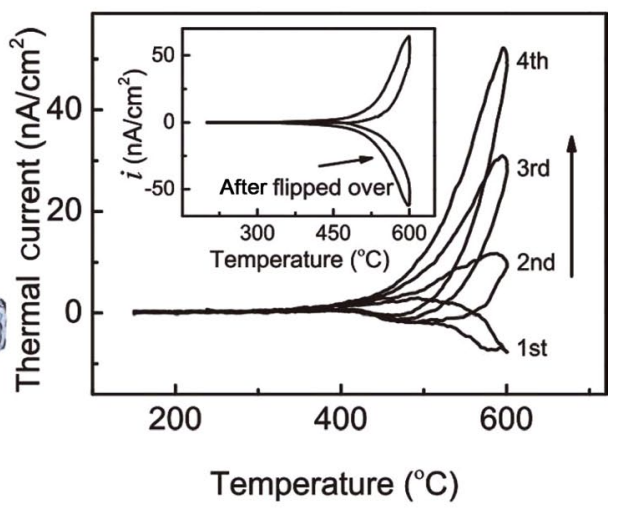

Fig. 13 (a) Bulk photovoltaic response of the $\mathrm{Bi}_{2} \mathrm{Te}_{3}$ film under different bending distances. The inset gives the photograph of the bending setup. Reproduced with permission from Ref. [161], (C) The Author(s) 2016. (b) Schematic view of the setup for verifying the flexo-photovoltaic effect. (c) The induced photocurrent density as a function of the loading force. (d) Positive photocurrent measured on a $\mathrm{TiO}_{2}(001)$ face with a $15 \mu \mathrm{N}$ force applied by the AFM tip. Reproduced with permission from Ref. [162], (C) The Authors 2018. (e) The diagram illustrates the distribution of carriers and electric dipoles in a strained $\mathrm{Bi}_{2} \mathrm{Te}_{3}$ film without thermal gradient and with a thermal gradient field. The thermoelectric field has an opposite direction with the flexoelectric field. Reproduced with permission from Ref. [164], (C) The Authors 2016. (f) Temperature dependence of the thermal currents of $\mathrm{Na}_{0.5} \mathrm{Bi}_{0.5} \mathrm{TiO}_{3}$-based ceramic plate with large internal field under three-point bending load. The inset shows the measured thermal currents of this ceramic plate without load. Reproduced with permission from Ref. [165], (C) American Physical Society 2018.

illumination were simultaneously applied onto some single crystals, as shown in Fig. 13(b). The result, as shown in Fig. 13(c), suggested that a significant photocurrent was induced by the applied mechanical force. Besides, the generation of such photocurrent was proved to be only originated from the flexophotovoltaic effect rather than other factor like Schottky contact. As shown in Fig. 13(d), the application of mechanical force can result in the sign of the short circuit current $I_{\mathrm{sc}}$ from negative to positive. Also, this flexo-photovoltaic effect can be improved very obviously when material dimension is decreased into the nanoscale. this study suggest that the application of flexoelectricity is effective route for improving the performance of solar cells and optoelectronic devices [163]. 
Recently, a newly reported flexo-caloric effect which defines as the strain gradient induced thermalcurrent has attracted considerable attentions. As suggested by Liu et al. [164], the flexoelectricity is proved to be responsible for the value of Seeback coefficient of some thermoelectric material like $\mathrm{BiFe}_{3}$. It is believed that the existence of flexoelectric diploes can effectively contribute to the thermoelectricity, as schematically illustrated in Fig. 13(e). Meanwhile, a remarkable flexo-caloric effect has been found in $\mathrm{Na}_{0.5} \mathrm{Bi}_{0.5} \mathrm{TiO}_{3}$-based ceramics [165], as shown in Fig. 13(f). Combined with the flexoelectric effect and electrocaloric effect, it is possible to design materials with strong thermo-electric coupling effect for sensing, thermal energy harvesting, or on-chip solid-state cooling applications [166].

\section{Outlook}

In summary, we reviewed the recent progress of flexoelectricity, mainly focused on the flexoelectric materials and their related applications. Flexoelectricity is not only limited to the dielectric materials, but also found to exist in the liquid crystals, bio-materials, and even semiconductors. The absence of symmetry constraint makes the flexoelectric materials suitable for most cases where non-uniform electric field distribution and non-uniform strain distribution exist. The recent discoveries utilized the flexoelectricity into many important application fields such as sensor and actuator, charge transportation, defect formation, domain tailoring, and some open applications like flexo-photovoltaic effect and flexo-caloric effect have been commented.

Although the study of flexoelectricity has an impressive achievement, the state-of-the-art understanding of this field is still in its initial stage. Lots of the fundamental problems regarding the flexoelectricity are unresolved. Herein we can only list parts of them. (1) Should the intrinsic flexoelectric coefficients of some kind of materials be beyond the theoretical value? Once it exists, the replacement of conventional electromechanical device by flexoelectric device may come true in the future. (2) The physical origin of the observed flexoelectricity in many perovskites is still unclear. Is there any universal rule to predict the flexoelectric coefficients in all kinds of the flexoelectric materials? (3) How to utilize the flexoelectricity into the low-symmetry crystals, especially for measuring the non-zero independent component of flexoelectric coefficients in those low-symmetry crystals? (4) Is there any way to induce large strain gradient, not just limited to the size effect?

Considering the past few years' progress in flexoelectric materials and related devices, the authors make the perspective as shown below.

1. Strain gradient is easier to exist in the complex material and flexible materials, resulting in the liquid crystals and bio-materials naturally suitable for flexoelectricity. Therefore, it is expected that the flexoelectricity will be widely used in liquid crystal display technology, bio-sensing, bio-medical, and bio-mimetic materials.

2. Due to the requirement of miniaturization in electronic devices, the role of flexoelectricity will draw more attentions. In the near future, micro/nano-scale flexoelectric sensing and actuating devices will be integrated into electronic devices. Among them, it is highly promising to find a lead-free and environmentally friendly flexoelectric material that is compatible for the AlN-based and silicon-based micro-electromechanical system.

3. Flexoelectricity is not limited by symmetry, giving it more space in material selection. In the future, it is expected to find a kind of natural material that is widely existed and has significantly enhanced flexoelectric properties.

\section{Acknowledgements}

This work was supported by the National Natural Science Foundation of China under Grant Nos. 11574126 and 11604135 , and partly by the Natural Science Foundation of Jiangxi Province (No. 20161BAB216110), China Postdoctoral Science Foundation (No. 2017M612162), and Postdoctoral Science Foundation of Jiangxi Province (No. 2017KY02).

\section{References}

[1] Lu H, Bark C-W, Esque de los Ojos D, et al. Mechanical writing of ferroelectric polarization. Science 2012, 336: 59-61.

[2] Catalan G, Lubk A, Vlooswijk AHG, et al. Flexoelectric rotation of polarization in ferroelectric thin films. Nat Mater 2011, 10: 963-967.

[3] Zubko P, Catalan G, Tagantsev AK. Flexoelectric effect in solids. Annu Rev Mater Res 2013, 43: 387-421. 
[4] Kalinin SV, Morozovska AN. Focusing light on flexoelectricity. Nat Nanotechnol 2015, 10: 916-917.

[5] Cross LE. Flexoelectric effects: Charge separation in insulating solids subjected to elastic strain gradients. $J$ Mater Sci 2006, 41: 53-63.

[6] Prost J, Pershan PS. Flexoelectricity in nematic and smectic-A liquid crystals. J Appl Phys 1976, 47: 2298-2312.

[7] Indenbom VL, Loginov EB, Osipov MA. Flexoelectric effect and crystal-structure. Kristallografiya 1981, 26: 1157-1162.

[8] Kogan SM. Piezoelectric effect during inhomogeneous deformation and acoustic scattering of carriers in crystals. Soviet Physics-Solid State 1964, 5: 2069-2070.

[9] Petrov AG. Flexoelectricity of model and living membranes. BBA-Biomembranes 2002, 1561: 1-25.

[10] Breneman KD, Brownell WE, Rabbitt RD. Hair cell bundles: Flexoelectric motors of the inner ear. PLoS One 2009, 4: e5201.

[11] Shu LL, Li F, Huang W, et al. Relationship between direct and converse flexoelectric coefficients. J Appl Phys 2014, 116: 144105.

[12] Shu LL, Wei XY, Pang T, et al. Symmetry of flexoelectric coefficients in crystalline medium. $J$ Appl Phys 2011, 110: 104106.

[13] Yudin PV, Tagantsev AK. Fundamentals of flexoelectricity in solids. Nanotechnology 2013, 24: 432001.

[14] Majdoub MS, Sharma P, Cagin T. Enhanced size-dependent piezoelectricity and elasticity in nanostructures due to the flexoelectric effect. Phys Rev B 2008, 77: 125424.

[15] Nguyen TD, Mao S, Yeh Y-W, et al. Nanoscale flexoelectricity. Adv Mater 2013, 25: 946-974.

[16] Chu K, Jang B-K, Sung JH, et al. Enhancement of the anisotropic photocurrent in ferroelectric oxides by strain gradients. Nat Nanotechnol 2015, 10: 972-979.

[17] Shu LL, Yong ZH, Jiang XN, et al. Flexoelectricity in low densification materials and its implication. $J$ Alloys Compd 2017, 695: 1555-1560.

[18] Fousek J, Cross LE, Litvin DB. Possible piezoelectric composites based on the flexoelectric effect. Mater Lett 1999, 39: 287-291.

[19] Tagantsev AK. Theory of flexoelectric effect in crystals. Zhurnal Eksperimental'noi i Teoreticheskoi Fiziki 1985, 88: $2108-2122$.

[20] Tagantsev AK. Piezoelectricity and flexoelectricity in crystalline dielectrics. Phys Rev B 1986, 34: 5883.

[21] Maranganti R, Sharma P. Atomistic determination of flexoelectric properties of crystalline dielectrics. Phys Rev B 2009, 80: 054109.

[22] Hong JW, Vanderbilt D. First-principles theory of frozen-ion flexoelectricity. Phys Rev B 2011, 84: 180101.

[23] Hong JW, Vanderbilt D. First-principles theory and calculation of flexoelectricity. Phys Rev B 2013, 88: 174107.
[24] Abdollahi A, Vásquez-Sancho F, Catalan G. Piezoelectric mimicry of flexoelectricity. Phys Rev Lett 2018, 121: 205502.

[25] Le Quang H, He QC. The number and types of all possible rotational symmetries for flexoelectric tensors. $P$ Roy Soc A-Math Phy 2011, 467: 2369-2386.

[26] Shu LL, Huang WB, Ryung Kwon S, et al. Converse flexoelectric coefficient $f_{1212}$ in bulk $\mathrm{Ba}_{0.67} \mathrm{Sr}_{0.33} \mathrm{TiO}_{3}$. Appl Phys Lett 2014, 104: 232902.

[27] Zhou H, Pei YM, Hong JW, et al. Analytical method to determine flexoelectric coupling coefficient at nanoscale. Appl Phys Lett 2016, 108: 101908.

[28] Hu TT, Deng Q, Liang X, et al. Measuring the flexoelectric coefficient of bulk barium titanate from a shock wave experiment. $J$ Appl Phys 2017, 122: 055106.

[29] Zhang SW, Liang X, Xu ML, et al. Shear flexoelectric response along 3121 direction in polyvinylidene fluoride. Appl Phys Lett 2015, 107: 142902.

[30] Zhang SW, Liu KY, Wu TH, et al. Experimental approach for measuring cylindrical flexoelectric coefficients. $J$ Appl Phys 2017, 122: 144103.

[31] Zhang SW, Liu KY, Xu ML, et al. Investigation of the 2312 flexoelectric coefficient component of polyvinylidene fluoride: Deduction, simulation, and mensuration. Sci Rep 2017, 7: 3134.

[32] Chu BJ, Salem DR. Flexoelectricity in several thermoplastic and thermosetting polymers. Appl Phys Lett 2012, 101: 103905.

[33] Chu BJ, Zhu WY, Li N, et al. Flexure mode flexoelectric piezoelectric composites. J Appl Phys 2009, 106: 104109.

[34] Zhou Y, Liu J, Hu XP, et al. Flexoelectric effect in PVDF-based polymers. IEEE Trans Dielect El In 2017, 24: 727-731.

[35] Ma WH, Cross LE. Flexoelectricity of barium titanate. Appl Phys Lett 2006, 88: 232902.

[36] Narvaez J, Saremi S, Hong JW, et al. Large flexoelectric anisotropy in paraelectric barium titanate. Phys Rev Lett 2015, 115: 037601.

[37] Ma WH, Cross LE. Flexoelectric polarization of barium strontium titanate in the paraelectric state. Appl Phys Lett 2002, 81: 3440-3442.

[38] Shu LL, Wang T, Jiang XN, et al. Verification of the flexoelectricity in barium strontium titanate through $\mathrm{d} 33$ meter. AIP Adv 2016, 6: 125003 .

[39] Li Y, Shu LL, Huang WB, et al. Giant flexoelectricity in $\mathrm{Ba}_{0.6} \mathrm{Sr}_{0.4} \mathrm{TiO}_{3} / \mathrm{Ni}_{0.8} \mathrm{Zn}_{0.2} \mathrm{Fe}_{2} \mathrm{O}_{4}$ composite. Appl Phys Lett 2014, 105: 162906.

[40] Shu LL, Wan MQ, Wang ZG, et al. Large flexoelectricity in $\mathrm{Al}_{2} \mathrm{O}_{3}$-doped $\mathrm{Ba}\left(\mathrm{Ti}_{0.85} \mathrm{Sn}_{0.15}\right) \mathrm{O}_{3}$ ceramics. Appl Phys Lett 2017, 110: 192903.

[41] Shu LL, Wei XY, Jin L, et al. Enhanced direct flexoelectricity in paraelectric phase of $\mathrm{Ba}\left(\mathrm{Ti}_{0.87} \mathrm{Sn}_{0.13}\right) \mathrm{O}_{3}$ ceramics. Appl Phys Lett 2013, 102: 152904.

[42] Ma WH, Cross LE. Strain-gradient-induced electric polarization in lead zirconate titanate ceramics. Appl 
Phys Lett 2003, 82: 3293-3295.

[43] Zhu JQ, Chen TW, Shu LL, et al. Flexoelectric fatigue in $(\mathrm{K}, \mathrm{Na}, \mathrm{Li})(\mathrm{Nb}, \mathrm{Sb}) \mathrm{O}_{3}$ ceramics. Appl Phys Lett 2018, 113: 182901.

[44] Shu LL, Li T, Wang ZG, et al. Flexoelectric behavior in PIN-PMN-PT single crystals over a wide temperature range. Appl Phys Lett 2017, 111: 162901.

[45] Ma WH, Cross LE. Observation of the flexoelectric effect in relaxor $\mathrm{Pb}\left(\mathrm{Mg}_{1 / 3} \mathrm{Nb}_{2 / 3}\right) \mathrm{O}_{3}$ ceramics. Appl Phys Lett 2001, 78: 2920-2921.

[46] Narvaez J, Catalan G. Origin of the enhanced flexoelectricity of relaxor ferroelectrics. Appl Phys Lett 2014, 104: 162903.

[47] Vales-Castro P, Roleder K, Zhao L, et al. Flexoelectricity in antiferroelectrics. Appl Phys Lett 2018, 113: 132903.

[48] Zubko P, Catalan G, Buckley A, et al. Straingradient-induced polarization in $\mathrm{SrTiO}_{3}$ single crystals. Phys Rev Lett 2007, 99: 167601.

[49] Biancoli A, Fancher CM, Jones JL, et al. Breaking of macroscopic centric symmetry in paraelectric phases of ferroelectric materials and implications for flexoelectricity. Nat Mater 2015, 14: 224-229.

[50] Borisevich AY, Eliseev EA, Morozovska AN, et al. Atomic-scale evolution of modulated phases at the ferroelectric-antiferroelectric morphotropic phase boundary controlled by flexoelectric interaction. Nat Commun 2012, 3: 775.

[51] Garten LM, Trolier-Mckinstry S. Enhanced flexoelectricity through residual ferroelectricity in barium strontium titanate. J Appl Phys 2015, 117: 094102.

[52] Resta R. Towards a bulk theory of flexoelectricity. Phys Rev Lett 2010, 105: 127601.

[53] Shu LL, Wan MQ, Jiang XN, et al. Frequency dispersion of flexoelectricity in PMN-PT single crystal. AIP $A d v$ 2017, 7: 015010.

[54] Zhang XT, Pan Q, Tian DX, et al. Large flexoelectriclike response from the spontaneously polarized surfaces in ferroelectric ceramics. Phys Rev Lett 2018, 121: 057602.

[55] Raphael RM, Popel AS, Brownell WE. A membrane bending model of outer hair cell electromotility. Biophys J 2000, 78: 2844-2862.

[56] Petrov AG. Flexoelectricity in lyotropics and in living liquid crystals. In: Flexoelectricity in Liquid Crystals. Imperial College Press, 2012: 177-210.

[57] Abou-Dakka M, Herrera-Valencia EE, Rey AD. Linear oscillatory dynamics of flexoelectric membranes embedded in viscoelastic media with applications to outer hair cells. J Non-Newton Fluid 2012, 185-186: 1-17.

[58] Jiang XN, Huang WB, Zhang SJ. Flexoelectric nano-generator: Materials, structures and devices. Nano Energy 2013, 2: 1079-1092.

[59] Kang HM, Hou ZD, Qin QH. Experimental study of time response of bending deformation of bone cantilevers in an electric field. J Mech Behav Biomed 2018, 77: 192-198.

[60] Williams WS, Breger L. Piezoelectricity in tendon and bone. J Biomech 1975, 8: 407-413.

[61] Vasquez-Sancho F, Abdollahi A, Damjanovic D, et al. Flexoelectricity in bones. Adv Mater 2018, 30: 1801413.

[62] Bayley H, Cronin B, Heron A, et al. Droplet interface bilayers. Mol BioSyst 2008, 4: 1191-1208.

[63] Booth MJ, Restrepo Schild V, Downs FG, et al. Functional aqueous droplet networks. Mol BioSyst 2017, 13: $1658-1691$.

[64] Challita EJ, Makhoul-Mansour MM, Freeman EC. Reconfiguring droplet interface bilayer networks through sacrificial membranes. Biomicrofluidics 2018, 12: 034112.

[65] Makhoul-Mansour M, Zhao WJ, Gay $\mathrm{N}$, et al. Ferrofluid-based droplet interface bilayer networks. Langmuir 2017, 33: 13000-13007.

[66] Freeman EC, Najem JS, Sukharev S, et al. The mechanoelectrical response of droplet interface bilayer membranes. Soft Matter 2016, 12: 3021-3031.

[67] Kancharala A, Freeman E, Philen M. A comprehensive flexoelectric model for droplet interface bilayers acting as sensors and energy harvesters. Smart Mater Struct 2016, 25: 104007.

[68] Tamaddoni N, Freeman EC, Sarles SA. Sensitivity and directionality of lipid bilayer mechanotransduction studied using a revised, highly durable membrane-based hair cell sensor. Smart Mater Struct 2015, 24: 065014.

[69] Trabi CL, Brown CV, Smith AAT, et al. Interferometric method for determining the sum of the flexoelectric coefficients $\left(e_{1}+e_{3}\right)$ in an ionic nematic material. Appl Phys Lett 2008, 92: 223509.

[70] Harden J, Mbanga B, Éber N, et al. Giant flexoelectricity of bent-core nematic liquid crystals. Phys Rev Lett 2006, 97: 157802 .

[71] Castles F, Morris SM, Coles HJ. The limits of flexoelectricity in liquid crystals. $A I P A d v$ 2011, 1: 032120.

[72] Buczkowska M, Derfel G. Influence of the surface pretilt angle on spatially periodic deformations in nematic layers. Liq Cryst 2018, 45: 961-964.

[73] Inoue Y, Hattori M, Moritake H. Thickness-independent dynamics in cholesteric liquid crystals. Opt Express 2017, 25: 3566-3577.

[74] Poddar A, Dhar J, Chakraborty S. Electro-osmosis of nematic liquid crystals under weak anchoring and second-order surface effects. Phys Rev E 2017, 96: 013114.

[75] Varanytsia A, Chien LC. Bimesogen-enhanced flexoelectrooptic behavior of polymer stabilized cholesteric liquid crystal. J Appl Phys 2016, 119: 014502.

[76] Varanytsia A, Chien LC. Giant flexoelectro-optic effect with liquid crystal dimer CB7CB. Sci Rep 2017, 7: 41333.

[77] Vaupotič N, Curk S, Osipov MA, et al. Short-range smectic fluctuations and the flexoelectric model of modulated nematic liquid crystals. Phys Rev E 2016, 93: 022704 . 
[78] Kim M, Jin HS, Lee SJ, et al. Liquid crystals for superior electro-optic performance display device with powersaving mode. Adv Opt Mater 2018, 6: 1800022.

[79] Lee D-J, Choi J-C, Park M-K, et al. Optical measurement of flexoelectric polarisation change in liquid crystals doped with bent-core molecules using hybrid-aligned structure. Liq Cryst 2017, 44: 1321-1331.

[80] Kim MS, Bos PJ, Kim D-W, et al. Field-symmetrization to solve luminance deviation between frames in a lowfrequency-driven fringe-field switching liquid crystal cell. Opt Express 2016, 24: 29568-29576.

[81] Kim M, Ham HG, Choi HS, et al. Flexoelectric in-plane switching (IPS) mode with ultra-high-transmittance, low-voltage, low-frequency, and a flicker-free image. $O p t$ Express 2017, 25: 5962-5971.

[82] Varshney D, Shriya S, Jain S, et al. Mechanically induced stiffening, thermally driven softening, and brittle nature of SiC. J Adv Ceram 2016, 5: 13-34.

[83] He JH, Hsin CL, Liu J, et al. Piezoelectric gated diode of a single $\mathrm{ZnO}$ nanowire. Adv Mater 2007, 19: 781-784.

[84] Ouyang J, Zhang W, Alpay SP, et al. Effect of elastic domains on electromechanical response of epitaxial ferroelectric films with a three-domain architecture. $J$ Adv Ceram 2013, 2: 1-10.

[85] Narvaez J, Vasquez-Sancho F, Catalan G. Enhanced flexoelectric-like response in oxide semiconductors. Nature 2016, 538: 219-221.

[86] Yurkov AS, Tagantsev AK. Strong surface effect on direct bulk flexoelectric response in solids. Appl Phys Lett 2016, 108: 022904.

[87] He B, Javvaji B, Zhuang XY. Size dependent flexoelectric and mechanical properties of barium titanate nanobelt: A molecular dynamics study. Physica B 2018, 545: 527-535.

[88] Qi L, Zhou SJ, Li AQ. Size-dependent bending of an electro-elastic bilayer nanobeam due to flexoelectricity and strain gradient elastic effect. Compos Struct 2016, 135: $167-175$.

[89] Shen SP, Hu SL. A theory of flexoelectricity with surface effect for elastic dielectrics. J Mech Phys Solids 2010, 58: 665-677.

[90] Huang WB, Kim K, Zhang SJ, et al. Scaling effect of flexoelectric $(\mathrm{Ba}, \mathrm{Sr}) \mathrm{TiO}_{3}$ microcantilevers. Phys Status Solidi RRL 2011, 5: 350-352.

[91] Bai G, Qin K, Xie QY, et al. Size dependent flexocaloric effect of paraelectric $\mathrm{Ba}_{0.67} \mathrm{Sr}_{0.33} \mathrm{TiO}_{3}$ nanostructures. Mater Lett 2017, 186: 146-150.

[92] Jeon BC, Lee D, Lee MH, et al. Flexoelectric effect in the reversal of self-polarization and associated changes in the electronic functional properties of $\mathrm{BiFeO}_{3}$ thin films. $A d v$ Mater 2013, 25: 5643-5649.

[93] Zhou WF, Chen P, Pan Q, et al. Lead-free metamaterials with enormous apparent piezoelectric response. $A d v$ Mater 2015, 27: 6349-6355.

[94] Lee D, Yoon A, Jang SY, et al. Giant flexoelectric effect in ferroelectric epitaxial thin films. Phys Rev Lett 2011, 107: 057602 .

[95] Gao P, Yang SZ, Ishikawa R, et al. Atomic-scale measurement of flexoelectric polarization at $\mathrm{SrTiO}_{3}$ dislocations. Phys Rev Lett 2018, 120: 267601.

[96] Tagantsev AK, Vaideeswaran K, Vakhrushev SB, et al. The origin of antiferroelectricity in $\mathrm{PbZrO}_{3}$. Nat Commun 2013, 4: 2229.

[97] Stengel M. Surface control of flexoelectricity. Phys Rev B 2014, 90: 201112.

[98] Mashkevich VS, Tolpygo KB. The interaction of vibrations of nonpolar crystals with electric fields. Soviet Physics Doklady 1957, 1: 690.

[99] Yudin PV, Ahluwalia R, Tagantsev AK. Upper bounds for flexoelectric coefficients in ferroelectrics. Appl Phys Lett 2014, 104: 082913.

[100] Yurkov AS, Tagantsev AK. Strong surface effect on direct bulk flexoelectric response in solids. Appl Phys Lett 2016, 108: 022904.

[101] Hong JW, Catalan G, Scott JF, et al. The flexoelectricity of barium and strontium titanates from first principles. $J$ Phys: Condens Matter 2010, 22: 112201.

[102] Banerjee AS, Suryanarayana P. Cyclic density functional theory: A route to the first principles simulation of bending in nanostructures. $J$ Mech Phys Solids 2016, 96: 605-631.

[103] Kvasov A, Tagantsev AK. Dynamic flexoelectric effect in perovskites from first-principles calculations. Phys Rev B 2015, 92: 054104 .

[104] Stengel M. Unified ab initio formulation of flexoelectricity and strain-gradient elasticity. Phys Rev $B$ 2016, 93: 245107.

[105] Dreyer CE, Stengel M, Vanderbilt D. Current-density implementation for calculating flexoelectric coefficients. Phys Rev B 2018, 98: 075153.

[106] Shi SH, Li P, Jin F. The mechanical analysis of thermomagneto-electric laminated composites in nanoscale with the consideration of surface and flexoelectric effects. Smart Mater Struct 2018, 27: 015018.

[107] Bendsøe MP, Kikuchi N. Generating optimal topologies in structural design using a homogenization method. Comput Method Appl M 1988, 71: 197-224.

[108] Wang MY, Wang XM, Guo DM. A level set method for structural topology optimization. Comput Method Appl M 2003, 192: 227-246

[109] Zhu J-H, Zhang W-H, Xia L. Topology optimization in aircraft and aerospace structures design. Arch Comput Method E 2016, 23: 595-622.

[110] Hong JW, Catalan G, Fang DN, et al. Topology of the polarization field in ferroelectric nanowires from first principles. Phys Rev B 2010, 81: 172101.

[111] Chae SC, Horibe Y, Jeong DY, et al. Evolution of the domain topology in a ferroelectric. Phys Rev Lett 2013, 110: 167601 .

[112] Ghasemi H, Park HS, Rabczuk T. A level-set based IGA 
formulation for topology optimization of flexoelectric materials. Comput Method Appl M 2017, 313: 239-258.

[113] Ghasemi H, Park HS, Rabczuk T. A multi-material level set-based topology optimization of flexoelectric composites. Comput Method Appl M 2018, 332: 47-62.

[114] Li Q, Nelson CT, Hsu SL, et al. Quantification of flexoelectricity in $\mathrm{PbTiO}_{3} / \mathrm{SrTiO}_{3}$ superlattice polar vortices using machine learning and phase-field modeling. Nat Commun 2017, 8: 1468.

[115] Kothari M, Cha M-H, Kim K-S. Critical curvature localization in graphene. I. Quantum-flexoelectricity effect. P Roy Soc A-Math Phy 2018, 474: 20180054.

[116] Mao YQ, Ai SG, Xiang XL, et al. Theory for dielectrics considering the direct and converse flexoelectric effects and its finite element implementation. Appl Math Model 2016, 40: 7115-7137.

[117] Wang Y-J, Li J, Zhu Y-L, et al. Phase-field modeling and electronic structural analysis of flexoelectric effect at $180^{\circ}$ domain walls in ferroelectric $\mathrm{PbTiO}_{3}$. J Appl Phys 2017, 122: 224101.

[118] Plymill A, Xu HX. Flexoelectricity in $\mathrm{ATiO}_{3}(\mathrm{~A}=\mathrm{Sr}, \mathrm{Ba}$, $\mathrm{Pb})$ perovskite oxide superlattices from density functional theory. $J$ Appl Phys 2018, 123: 144101.

[119] Nanthakumar SS, Zhuang XY, Park HS, et al. Topology optimization of flexoelectric structures. $J$ Mech Phys Solids 2017, 105: 217-234.

[120] Deng F, Deng Q, Shen SP. A three-dimensional mixed finite element for flexoelectricity. $J$ Appl Mech 2018, 85 : 031009.

[121] Wan MQ, Yong ZH, Huang WB, et al. Design of a flexure composite with large flexoelectricity. J Mater Sci: Mater El 2017, 28: 6505-6511.

[122] Jiang L, Xu X, Zhou Y. Interrelationship between flexoelectricity and strain gradient elasticity in ferroelectric nanofilms: A phase field study. J Appl Phys 2016, 120: 234102.

[123] Xu XF, Jiang LM, Zhou YC. Reduction of leakage currents in ferroelectric thin films by flexoelectricity: A phase field study. Smart Mater Struct 2017, 26: 115024.

[124] Hamdia KM, Ghasemi H, Zhuang XY, et al. Sensitivity and uncertainty analysis for flexoelectric nanostructures. Comput Method Appl M 2018, 337: 95-109.

[125] Yadav AK, Nelson CT, Hsu SL, et al. Observation of polar vortices in oxide superlattices. Nature 2016, 530: 198-201.

[126] Mo C, Davidson J, Clark WW. Energy harvesting with piezoelectric circular membrane under pressure loading. Smart Mater Struct 2014, 23: 045005.

[127] Azizi S, Ghodsi A, Jafari H, et al. A conceptual study on the dynamics of a piezoelectric MEMS (Micro Electro Mechanical System) energy harvester. Energy 2016, 96: 495-506.

[128] Jasim A, Wang H, Yesner G, et al. Optimized design of layered bridge transducer for piezoelectric energy harvesting from roadway. Energy 2017, 141: 1133-1145.
[129] Ravi S, Zilian A. Monolithic modeling and finite element analysis of piezoelectric energy harvesters. Acta Mech 2017, 228: 2251-2267.

[130] Wong VK, Ho JH, Chai AB. Performance of a piezoelectric energy harvester in actual rain. Energy 2017, 124: 364-371.

[131] Yang ZB, Wang YQ, Zuo L, et al. Introducing arc-shaped piezoelectric elements into energy harvesters. Energy Convers Manag 2017, 148: 260-266.

[132] Zhou ZY, Qin WY, Zhu P. Harvesting acoustic energy by coherence resonance of a bi-stable piezoelectric harvester. Energy 2017, 126: 527-534.

[133] Qi L, Huang SJ, Fu GY, et al. On the mechanics of curved flexoelectric microbeams. Int J Eng Sci 2018, 124: 1-15.

[134] Yan Z. Modeling of a piezoelectric/piezomagnetic nano energy harvester based on two dimensional theory. Smart Mater Struct 2018, 27: 015016.

[135] Liang X, Hu SL, Shen SP. Nanoscale mechanical energy harvesting using piezoelectricity and flexoelectricity. Smart Mater Struct 2017, 26: 035050.

[136] Wang KF, Wang BL. Energy gathering performance of micro/nanoscale circular energy harvesters based on flexoelectric effect. Energy 2018, 149: 597-606.

[137] Wang KF, Wang BL. An analytical model for nanoscale unimorph piezoelectric energy harvesters with flexoelectric effect. Compos Struct 2016, 153: 253-261.

[138] Choi S-B, Kim G-W. Measurement of flexoelectric response in polyvinylidene fluoride films for piezoelectric vibration energy harvesters. J Phys D: Appl Phys 2017, 50: 075502 .

[139] Han JK, Jeon DH, Cho SY, et al. Nanogenerators consisting of direct-grown piezoelectrics on multi-walled carbon nanotubes using flexoelectric effects. Sci Rep 2016, 6: 29562.

[140] Zhu RJ, Wang ZM, Ma H, et al. Poling-free energy harvesters based on robust self-poled ferroelectric fibers. Nano Energy 2018, 50: 97-105.

[141] Ray MC. Analysis of smart nanobeams integrated with a flexoelectric nano actuator layer. Smart Mater Struct 2016, 25: 055011.

[142] Ray MC. Mesh free model of nanobeam integrated with a flexoelectric actuator layer. Compos Struct 2017, 159: 63-71.

[143] Zhang SW, Liu KY, Xu ML, et al. A curved resonant flexoelectric actuator. Appl Phys Lett 2017, 111: 082904.

[144] Bhaskar UK, Banerjee N, Abdollahi A, et al. A flexoelectric microelectromechanical system on silicon. Nat Nanotechnol 2016, 11: 263-266.

[145] Kwon SR, Huang WB, Zhang SJ, et al. Flexoelectric sensing using a multilayered barium strontium titanate structure. Smart Mater Struct 2013, 22: 115017.

[146] Huang WB, Yan X, Kwon SR, et al. Flexoelectric strain gradient detection using $\mathrm{Ba}_{0.64} \mathrm{Sr}_{0.36} \mathrm{TiO}_{3}$ for sensing. Appl Phys Lett 2012, 101: 252903.

[147] Huang W, Kwon SR, Yuan FG, et al. A flexoelectric 
micro-accelerometer. ASME 2012, 9: 597-603.

[148] Yan X, Huang WB, Kwon SR, et al. Design of a curvature sensor using a flexoelectric material. In: Proceedings of the SPIE 8692, Sensors and Smart Structures Technologies for Civil, Mechanical, and Aerospace Systems, 2013: 86920N.

[149] Merupo VI, Guiffard B, Seveno R, et al. Flexoelectric response in soft polyurethane films and their use for large curvature sensing. J Appl Phys 2017, 122: 144101.

[150] Kwon SR, Huang WB, Zhang SJ, et al. Study on a flexoelectric microphone using barium strontium titanate. J Micromech Microeng 2016, 26: 045001.

[151] Gómez A, Vila-Fungueiriño JM, Moalla R, et al. Electric and mechanical switching of ferroelectric and resistive states in semiconducting $\mathrm{BaTiO}_{3-\delta}$ films on silicon. Small 2017, 13: 1701614.

[152] Lu HD, Liu S, Ye ZY, et al. Asymmetry in mechanical polarization switching. Appl Phys Lett 2017, 110: 222903.

[153] Guo R, Shen L, Wang H, et al. Tailoring self-polarization of $\mathrm{BaTiO}_{3}$ thin films by interface engineering and flexoelectric effect. Adv Mater Interfaces 2016, 3: 1600737.

[154] Vorotiahin IS, Morozovska AN, Eliseev EA, et al. Flexocoupling impact on the kinetics of polarization reversal. Phys Rev B 2017, 95: 014104.

[155] Park SM, Wang B, Das S, et al. Selective control of multiple ferroelectric switching pathways using a trailing flexoelectric field. Nat Nanotechnol 2018, 13: 366-370.

[156] Seol D, Yang SM, Jesse S, et al. Dynamic mechanical control of local vacancies in $\mathrm{NiO}$ thin films. Nanotechnology 2018, 29: 275709.

[157] Das S, Wang B, Cao Y, et al. Controlled manipulation of oxygen vacancies using nanoscale flexoelectricity. Nat Commun 2017, 8: 615.

[158] Eliseev EA, Morozovska AN, Glinchuk MD, et al. Lost surface waves in nonpiezoelectric solids. Phys Rev B 2017, 96: 045411.

[159] Yang WJ, Deng Q, Liang X, et al. Lamb wave propagation with flexoelectricity and strain gradient elasticity considered. Smart Mater Struct 2018, 27: 085003.

[160] Morozovska AN, Glinchuk MD, Eliseev EA, et al. Flexocoupling-induced soft acoustic modes and the spatially modulated phases in ferroelectrics. Phys Rev B 2017, 96: 094111.

[161] Liu YC, Chen JD, Wang C, et al. Bulk photovoltaic effect at infrared wavelength in strained $\mathrm{Bi}_{2} \mathrm{Te}_{3}$ films. Apl Mater 2016, 4: 126104.

[162] Yang MM, Kim DJ, Alexe M. Flexo-photovoltaic effect. Science 2018, 360: 904-907.

[163] Lee W, Kim D, Lim J, et al. Light-induced electrical switch via photo-responsive nanocomposite film. Sensor Actuat B Chem 2018, 266: 724-729.

[164] Liu YC, Chen JD, Deng HY, et al. Anomalous thermoelectricity in strained $\mathrm{Bi}_{2} \mathrm{Te}_{3}$ films. Sci Rep 2016, 6: 32661.

[165] Chen P, Zhang HF, Chu BJ. Strain gradient induced thermal-electrical response in paraelectric $\mathrm{Na}_{0.5} \mathrm{Bi}_{0.5} \mathrm{TiO}_{3-}$ based ceramics. Phys Rev Materials 2018, 2: 034401.

[166] Patel S, Chauhan A, Madhar NA, et al. Flexoelectric induced caloric effect in truncated Pyramid shaped $\mathrm{Ba}_{0.67} \mathrm{Sr}_{0.33} \mathrm{TiO}_{3}$ ferroelectric material. J Electron Mater 2017, 46: 4166-4171.

Open Access This article is licensed under a Creative Commons Attribution 4.0 International License, which permits use, sharing, adaptation, distribution and reproduction in any medium or format, as long as you give appropriate credit to the original author(s) and the source, provide a link to the Creative Commons licence, and indicate if changes were made.

The images or other third party material in this article are included in the article's Creative Commons licence, unless indicated otherwise in a credit line to the material. If material is not included in the article's Creative Commons licence and your intended use is not permitted by statutory regulation or exceeds the permitted use, you will need to obtain permission directly from the copyright holder.

To view a copy of this licence, visit http://creativecommons. org/licenses/by/4.0/. 